\title{
Robust CFAR Detector Based on Truncated Statistics for Polarimetric Synthetic Aperture Radar
}

Tao Liu, Ziyuan Yang, Armando Marino, Member, IEEE, Gui Gao, Member, IEEE, and Jian Yang, Senior Member, IEEE

\begin{abstract}
Constant false alarm rate (CFAR) algorithms using local training window are widely used for ship detection with synthetic aperture radar (SAR) imagery. However, when the density of targets is high, such as in busy shipping lines and crowded harbors, the background statistics may be contaminated by the presence of nearby targets in the training window. Recently a robust CFAR detector based on truncated statistics (TS) was proposed. However, the truncation of data in the format of polarimetric covariance matrices is much more complicated with respect to the truncation of intensity (single polarization) data. In this paper a polarimetric truncated statistic CFAR (PWF-TS-CFAR) is proposed to estimate the background parameters accurately in the contaminated sea clutter for Pol SAR imagery. The CFAR detector uses polarimetric whitening filter (PWF) to turn the multidimensional problem to a one dimensional case. It uses the truncation to exclude possible statistically interfering outliers and uses TS to model the remaining background samples. The algorithm does not require a prior knowledge of the interfering targets and it is performed iteratively and adaptively to derive better estimates of the polarimetric covariance matrix (although this is computationally expensive). The PWF-TS-CFAR detector provides accurate background clutter modeling, a stable false alarm property, and improves detection performance in high-target-density situations. RadarSat2 data are used to verify our derivations and the results are in line with the theory.
\end{abstract}

Index Terms-Constant false alarm rate (CFAR), Sea clutter, Statistical modeling, Synthetic aperture radar (SAR), Ship detection, Truncated statistics (TS), Polarimetric Whitening Filter (PWF)

This work was supported in part by the National Natural Science Foundation of China under Project 61771483, 41822105, 61490693, Key Research Plan of Hunan Province under Project 2019SK2173, and the Field Foundation under Project 61404160109.

T. Liu and Ziyuan Yang are with the school of electronic engineering, Naval University of Engineering, Wuhan, 430033, China (e-mail:

liutao1018(a,hotmail.com).

A. Marino is with the Faculty of Natural Sciences, University of Stirling, UK (armando.marino@stir.ac.uk).

G. Gao is with the Faculty of Geoscience and Environmental Engineering, Southwest Jiaotong University, Chengdu, 611756, China, and the College of Traffic Engineering, Hunan University of Technology, Zhuzhou, 412007,

China with (e-mail: dellar@126.com).

J. Yang is with the department of electronic engineering, Tsinghua University, Beijing, 100084, China (e-mail:yangjian_ee@tsinghua.edu.cn).

\section{INTRODUCTION}

$P$ OLARIMETRIC Synthetic Aperture Radar (PolSAR) is a multi-dimension remote sensing system which has been largely used for marine surveillance, including ship detection [1]. The current challenges in ship detection mostly lie in two aspects: the first is to detect small ships densely packed in inshore regions while the second is to detect ships during the medium or high sea states. This manuscript is concerned with the first challenge.

\section{A. Review of Ship Detection in PolSAR Imagery}

In SAR images, a common feature of ships is a relatively large backscattering signal, which is usually brighter than the sea background. This led to designing algorithms that detect vessels using a statistical test on the intensity of the sea clutter [1]. If the statistical distributions of clutter and targets are known, an optimal detector can be designed via the likelihood ratio test (LRT). The optimal polarimetric detector (OPD) was proposed by Novak et al. [2]. Polarimetric decompositions and other physical based models [3] have also been adopted for ship detection in PolSAR imagery. Ringrose used the Cameron decomposition method to detect ships from SIR-C data [4]. Chen $e t a l$. proposed a polarization cross entropy for detecting ships [5]. Zhang et al. used the space relation characteristics of ships to detection targets [6]. It has been shown that ship wakes can help detect ships and also provide useful information about heading and velocity [7]-[10]. However, ship wake detection is influenced by various factors, such as radar frequency, angle of view and angle of incidence and sea state.

The statistical distribution or characteristics of ship backscattering is difficult to obtain, since this depends highly on the physical characteristics of the vessels themselves. Recently, a Polarimetric Notch Filter (PNF) was proposed that tries to separate ships and vessels based on their polarimetric behavior [11]-[12]. It does not assume a prior information about the ships and can provide good performance by minimizing the sea clutter power [1][13]-[14]. Another filter working in the absence of ship prior information is the Polarimetric Whitening Filter (PWF) proposed by Novak $[15][16]$. The PWF utilizes the polarimetric information to minimize the statistical variation due to speckle. In early experiments, its performance has been proved to be the closest to that of the OPD [15][16]. The PWF combines all scattering vector correlation information into a single quantity, the scattering matrix. This matrix is not adequate for representation 
of the filtered scene received wave, which should be partially polarized because of the spatial scene signal variations within the processing window. The Novak's PWF filter might be used to facilitate image interpretation, but results obtained should be considered with caution because of the eventual loss of polarimetric information [17].The PWF was further extended to Multi-look Polarimetric Whitening Filter (MPWF) in terms of a polarimetric covariance matrix by Liu and Lopes et al. [18]-[19], which makes it consistent with the principle of speckle filtering introduced by Touzi et al.[17]. Since the MPWF and the PWF share the same theoretical background in this paper when we refer to PWF we consider both of them depending if we perform or not averaging.

Both the PNF and PWF are based on the polarimetric covariance matrix, but Touzi et al. showed that the covariance matrix and the tools based on it such as PNF and PWF detectors cannot cover the full polarimetric information required for enhanced ship detection [20]. Touzi et al. also showed that excursion of the degree of polarization provide important information that is not exploited by the covariance matrix as well as the Marino detector [21]. The degree of polarization provides very important information that is not covered by conventional covariance matrix optimization tools such as the Notch detector.

Since we do not have priori information about ships, the test is set on the clutter distribution and therefore it considers the probability of false alarms (PFA). The constant false alarm rate (CFAR) tries to keep PFA constant by selecting a suitable threshold that adapts with the background clutter [1]. To adapt the threshold an accurate modeling and estimation of the statistical distribution of local background clutter is generally done using a sliding window [1][13]-[14]. Different statistical models are used to fit heterogeneous sea clutter [22]. In practice, in high-target-density situations such as busy shipping lines and crowded harbors the training area used to estimate clutter characteristics are generally contaminated by the presence of other targets [23]. This problem was already solved in single polarization case [23], but there is still no solution when using PolSAR data. The aim of this paper is to derive a PolSAR CFAR algorithm which is robust against statistical contamination in the training window. After the target contamination is removed, a statistically robust methodology is presented to model the remaining sea clutter pixels. The PWF is used to produce the image that is truncated. In the following, a brief review of CFAR detectors in contaminated background is presented.

\section{B. Survey of CFAR detectors in the Contaminated Surroundings}

\section{1) Single polarization case}

The benchmark of CFAR algorithms is the traditional cell-averaging CFAR (CA-CFAR) which averages each pixel inside the reference window [24]. This is appropriate for homogeneous clutter but it is affected by contamination.

Data ranking or censoring with different restrictions can be used to remove outliers. The greatest-of CFAR (GO-CFAR) [25] and the smallest-of CFAR (SO-CFAR) [26] detectors are both variations of CA-CFAR, which split the reference window in spatial subset before averaging. The GO-CFAR trains on the clutter in the greatest subset while the SO-CFAR uses the smallest. On the other hand, the variability index CFAR (VI-CFAR) [27] chooses the particular group of reference pixels adaptively to estimate background statistics. VI-CFAR is expected to degrade performance when the distribution of the clutter is complicated and it cannot be modeled by a simple spatial division. Another well-known CFAR algorithm is the ordered statistic CFAR (OS-CFAR) which deals with outliers within the reference window [28]. The OS-CFAR keeps a significantly higher detection rate and low false alarm rate [29]. The OS-CFAR disadvantages are generating a small loss in detection rate in homogeneous clutter compared with the CA-CFAR and a high computational cost. As a generalization of the OS-CFAR detector, the trimmed mean CFAR (TM-CFAR) [30] estimates distribution parameters by the mean of a set of rank-ordered values. In addition, the censored mean-level detector (CMLD) [31] is designed to obtain acceptable performance in the presence of interfering targets, which is adaptive to several clutter environments. Both ranking and censoring techniques are employed in the CMLD. However, the CMLD may lose its robustness and degrade its CFAR properties when a prior knowledge of the interfering targets is absent [32].

The last proposed algorithm is the truncated statistic CFAR (TS-CFAR) detector, which is also the base of this work. If the pixel intensity is higher than a specified threshold, the pixel will be excluded from the training samples and truncated data are used to estimate the clutter parameters. This involves using different probability density function (PDF) when modeling the truncated data. The difference between truncation and censoring is whether the number of the removed pixels is recorded or not. The number of truncated data points remains unknown, while the number of censored data points is stored. When it comes to model the background in a statistically rigorous manner, the distinction turns to be important [23]. In particular, the truncated data can be represented by truncated versions of the gamma distribution in the single polarization case, which was revealed to be robust for single polarization images [23].

\section{2) Full polarization case}

Commonly that the problem of contamination for PolSAR data was addressed using two steps. A) We select a clean patch close to the detection region and use this to estimate clutter parameters; B) We perform a CFAR using those parameters. This does not assure us the absence of ships in the training region. However, if ships are few and the region large, the effect of contamination on the statistical model can be ignored as seen in Gao et al. [14]. This procedure requires supervision. Additionally, if the number of outliers is large or if the sea clutter is too heterogeneous, this procedure may fail.

For polarimetric data, the problem of removing contamination in local window is still open, since the truncation can only be applied to a $1 \mathrm{D}$ array and not a $3 \mathrm{D}$ one. A humble idea is to process the different polarimetric SAR channels independently and combine them at the end. This will result in losing polarimetric information since the complex correlation between channels will be ignored.

In this paper, we propose a solution based on the PWF. The PWF is transforming a matrix into an intensity [15][16] which is formally equivalent to using a single channel. 


\section{Contributions of this study}

The statistics of the PWF in Wishart case have already been derived [22][33]. Here we will extend this to the truncated expression. The more challenging issue is to accurately estimate the original polarimetric covariance matrix starting from the truncated data. In other words, we need to find a relationship between the covariance matrix after truncation and the true covariance matrix. The starting point is the relation between the second moments of a truncated vector and the true covariance matrix derived by G. M. Tallis [35]. Unfortunately this relation assumes the vector should be real. In this work we have extended the theorem to the complex case and multilook case. The proof is presented in the Appendix.

In details, the truncated gamma distribution and the elliptically truncated Wishart distribution are proposed to model the truncated PWF samples. A statistically rigorous approach is adopted to handle the truncated data in the PWF-TS-CFAR. We use the assumption that the speckle is fully developed and that the radar cross section is locally constant, which makes the problem mathematically tractable. It is well known that this assumption may not be sustained as the SAR resolution increases and the sea state roughens. Fortunately, this assumption should cover the most of the high-target-density situations, which mostly happen in sheltered areas (e.g. harbors). In addition, a mixture of several gamma distributions can also be used to model large scale heterogeneity [36]. Finally, since the PWF distribution was found out to be a gamma and this is a special case of the generalized gamma distribution ( GГD), we decide to improve the robustness of the statistical model by add an expression which considers the GГD .

This paper is organized as follows. Section II presents the statistical of PolSAR data involving the MPWF. Section III provides an introduction to TS for a matrix and derives the proposed TS-CFAR detectors for Pol-SAR measurements. In addition, the performance of parameter estimation and the false alarm regulation property and the receiver operating characteristic (ROC) of the CFAR detector are examined based on simulated measurements of sea clutter drawn from the truncated Wishart distribution. In section IV, an adaptive truncated threshold is presented, and the PWF-TS-CFAR detector is compared among different parameter estimators via real Radarsat-2 SAR measurements. Finally, Section V presents the main conclusions and perspectives.

\section{Statistical Model OF POL-SAR DATA}

\section{A. Description of Pol-SAR Data}

Under far-field hypothesis, the scattering characteristics of a target is represented by a polarimetric scattering matrix, expressed in Eq. (1) when a Horizontal-Vertical linear basis is used [1][15]

$$
\mathbf{S}=\left[\begin{array}{ll}
S_{H H} & S_{H V} \\
S_{V H} & S_{V V}
\end{array}\right]
$$

where $S_{x y}$ represents the complex scattering coefficient with $x$ standing for the transmitting polarization, and $y$ for the receiving polarization (H-horizontal polarization, V-vertical polarization). When the system is mono-static and the reciprocity limitation is satisfied, we have $S_{H V}=S_{V H}$. Then the scattering vector can be defined as

$$
\mathbf{k}=\left[\begin{array}{c}
S_{H H} \\
\sqrt{2} S_{H V} \\
S_{V V}
\end{array}\right]
$$

To reduce speckle in a SAR image, filtering or multi-look processing can be used. We can therefore obtain the covariance matrix as [15][19]

$$
\mathbf{C}=\frac{1}{L} \sum_{i=1}^{L} \mathbf{k}_{i} \mathbf{k}_{i}^{\dagger}
$$

where $L$ is the number of averaged looks. The superscript $\dagger$ denotes conjugate transpose. The multilook covariance matrix $\mathbf{C}$ is a random variable and therefore it can be modeled using a PDF. $\mathbf{\Sigma}=\mathrm{E}\{\mathbf{C}\}$ is the covariance matrix of the speckle in the Gaussian case, $\boldsymbol{\Sigma}$ is the statistical mean of the multilook covariance matrix $\mathbf{C}$, and $\mathrm{E}(\bullet)$ represents the expectation operator.

One part of the SAR pixel variation is due to the interference between scatterers within a resolution cell, which is also referred to as speckle. The other part of the variation is due to the fluctuation of the radar cross section (RCS), which is also referred to as texture [14]. The variation generated by texture is slower than the one generated by speckle. In another word, though the texture is modulated, it can only be observed on a large amount of pixels. The PDF of a covariance matrix without texture is a Wishart distribution based on multivariate complex Gaussian distribution, which models pure and fully developed speckle [37][38]

$$
f_{C}(\mathbf{C})=\frac{L^{L d}|\mathbf{C}|^{L-d} \exp \left(-L \operatorname{Tr}\left(\boldsymbol{\Sigma}^{-1} \mathbf{C}\right)\right)}{\Gamma_{d}(L)|\mathbf{\Sigma}|^{L}}
$$

where $L$ is the number of looks, $d$ is the dimension of $\mathbf{C}$, $\operatorname{Tr}(\bullet)$ is the trace operator, $\Gamma(\bullet)$ is the gamma function and $\Gamma_{d}(L)$ is:

$$
\Gamma_{d}(L)=\pi^{\frac{1}{2} d(d-1)} \Gamma(L) \cdots \Gamma(L-d+1)
$$

\section{B. Statistics of the MPWF}

The different polarimetric channels can be processed together to reduce speckle [19]. Novak and Burl [15] proposed the polarimetric whitening filter (PWF) which is an optimization aimed at maximally reducing speckle. Novak et al. [16] later showed that the PWF can improve the target detection performance. The PWF was further enhanced into a multi-look polarimetric whitening filter (MPWF) by Lopes and Liu et al. [18]-[19]. PWF can be seen as a generalization of the MPWF.

The expression of the MPWF for L looks is [15]:

$$
z=\frac{1}{L} \sum_{i=1}^{L} \mathbf{k}_{i}^{\dagger} \boldsymbol{\Sigma}^{-1} \mathbf{k}_{i}=\operatorname{tr}\left(\boldsymbol{\Sigma}^{-1} \mathbf{C}\right)
$$

where $\operatorname{tr}(\bullet)$ is the trace operator and $L$ as the number of looks.

When speckle is fully developed as mentioned in section A, $z$ obeys a Gamma distribution after a series of mathematical deduction [33][34]: 


$$
z \sim \gamma\left(L d, \frac{1}{L}\right)
$$

where $\gamma(\alpha, \beta)$ denotes a Gamma distribution with shape parameter $\alpha$ and scale parameter $\beta . \gamma(\alpha, \beta)$ can be represented as follows [39]

$$
\gamma(\alpha, \beta)=\frac{1}{\Gamma(\alpha)} \frac{1}{\beta}\left(\frac{x}{\beta}\right)^{\alpha-1} \exp \left(-\frac{x}{\beta}\right)
$$

where $\Gamma(a)=\int_{0}^{\infty} y^{a-1} e^{-y} d y$ is the gamma function.

\section{Polarimetric TRUnCATED Statistics CFAR Detector}

\section{A. Truncated Statistics}

Clutter parameter estimation in areas contaminated by outliers (or interfering targets) is challenging. In this work we will use truncation since it allows deriving a rigorous statistical model.

The PDF of the truncated data can be calculated starting from the PDF of the untruncated data.

Let's assume the random variable $z$ can be modeled by the probability density function (PDF) $f_{z}(z)$ and a cumulative distribution function (CDF) $F_{z}(z)$. Suppose $\tilde{z}$ be the truncated version of $z$ after the truncation by a threshold $\rho$. The right-truncated distribution can be defined as

$$
f_{\tilde{z}}(z ; \rho)=f_{z}(z \mid z<\rho)= \begin{cases}\frac{f_{z}(z)}{F_{z}(\rho)}, & 0<z \leq \rho \\ 0, & x>\rho\end{cases}
$$

The division by $F_{z}(\rho)$ ensures that the integral of $f_{\tilde{z}}(z ; \rho)$ is unitary. The truncation threshold is also refereed to truncation depth. Since the priori knowledge about outliers is unknown, it is difficult to estimate the optimal truncation depth. A lower threshold $\rho$ returns a higher probability to exclude all outliers; however, if the truncation threshold is too excessive, inaccurate parameter estimation may occur.

There are several studies that deal with the one dimensional case [40][41]. The first results on truncated moments considered a linear truncated multivariate normal (MVN) distribution [42]. Tallis later extended his results of linear truncations to the case of elliptical and radial truncation [35]. Tallis [43] built on previous results to compute the moments of a normal distribution with a plane truncation. For a review of truncated moments for different continuous distributions, see [29][45]. The constraint on the elliptical and radial truncation is as follows [35]:

$$
0 \leq z=x^{\prime} \boldsymbol{\Sigma}^{-1} x \leq \rho
$$

where $x$ is a multivariate vector, ' is the transpose operator, and $z, \Sigma, \rho$ are all real.

Interestingly, this constraint is also the form of the PWF (Eq (6)) when the number of look is $L=1$. This further motivated our work.

\section{B. PWF-TS-CFAR Detector}

In this section, CFAR detectors based on TS are derived for SLC and MLC SAR measurements, which are modeled by the gamma and Wishart distributions. We consider the MLC case first, since the SLC can be seen as a special case of MLC. The intensity of PWF pixels is positive and assumed to be independent and identically distributed.

\section{1) Estimation of the Polarimetric covariance matrix}

The complete and truncated distributions (Eq (7) and (9)) model the PWF properly only in the assumption that the polarimetric covariance matrix is estimated accurately. The problem of estimating the polarimetric covariance matrix from the truncated data is of paramount importance.

A mentioned previously the MPWF is an elliptical and radial truncation. For fully developed speckle (i.e. Wishart distribution), the true covariance matrix can be derived using the second truncated moments. From Tallis' paper, it turns out that [35]

$$
\mathfrak{S}(\rho ; \boldsymbol{\Sigma})=\frac{\boldsymbol{\Sigma}}{\mu_{T}(\rho)}, \mu_{T}(\rho) \equiv \frac{F_{d}(\rho)}{F_{d+2}(\rho)}
$$

with $F_{v}(\bullet)$ denoting the cumulative distribution of a $\chi^{2}$ variable with $d$ degrees of freedom. Inverting (18) so as to express $\boldsymbol{\Sigma}$ as a function of $\mathfrak{S}$ is trivial, since $c_{T}(\rho)$ is a scalar damping factor independent of $\boldsymbol{\Sigma}$. This result is only valid for Gaussian distributed vectors or covariance matrices.

According to the methods in [35][48], and the relations between the $\chi^{2}$ cumulative distribution $F_{v}(\bullet)$ and the incomplete gamma function $\Gamma(a, b)[39]$, we can extended the real number results to the complex and multilook case (Appendix A)

$$
\boldsymbol{\Sigma}=\mu_{T}(\rho) \mathfrak{S}(\rho ; \boldsymbol{\Sigma}), \mu_{T}(\rho) \equiv \frac{\Gamma(L d, L \rho)}{\Gamma(L d+1, L \rho)}
$$

with $\Gamma(a, b)=\frac{1}{\Gamma(a)} \int_{0}^{b} y^{a-1} e^{-y} d y$. Thus we can estimate the true polarimetric covariance matrix of the clutter from the second moments of the truncated data. This makes the PDF in Eq (12) more accurate.

\section{2) Estimation of the Equivalent Number of Looks}

From Eq (12) we can see that the difference between the covariance matrix of the truncated data and the true covariance matrix is just a coefficient. The polarimetric behavior of the clutter is unchanged between the complete and truncated set after the elliptical and radial truncation (PWF). It is clear that if we don't adjust the covariance matrix with the correct coefficient $\mu_{T}(\rho)$, the output of the MPWF will be different in scale. This difference only affects the mean of the MPWF distribution. Therefore there are two parameters that need to be estimated, the equivalent number of looks (ENL) and the mean of the MPWF. The distribution of the MPWF without covariance truncation is

$$
z \sim \gamma\left(L d, \frac{1}{L}\right)
$$

Its cumulative distribution function $(\mathrm{CDF})$ is

$$
F_{z}(z ; L, d)=\Gamma(L d, L z)
$$

The PDF of $\tilde{z}$, the truncated data is 


$$
f_{\tilde{z}}(z ; L, d)=\left\{\begin{array}{l}
(L)^{L d} \frac{z^{L d-1} \exp (-L z)}{\Gamma(L d) \Gamma(L d, L \rho)}, 0<z \leq \rho \\
0, \quad z>\rho .
\end{array}\right.
$$

The other estimator is based on the moments. The $\mathrm{n}$-th order moments of the truncated MPWF in Eq (15) is

$$
E_{\tilde{z}}\left(z^{n}\right)=\frac{\Gamma(L d+n) \Gamma(L d+n, L \rho)}{L^{n} \Gamma(L d) \Gamma(L d, L \rho)}
$$

Then we can use the first moments of the truncated data to estimate the ENL:

$$
\Gamma(x+1)=x \Gamma(x)
$$

We can get

$$
\langle z\rangle=\frac{d \Gamma(\hat{L} d+1, \hat{L} \rho)}{\Gamma(\hat{L} d, \hat{L} \rho)}
$$

A maximum likelihood (ML) estimator for the ENL can be also obtained from the likelihood function

$$
\begin{aligned}
& \mathcal{L}(L \mid \tilde{z})=\prod_{i=1}^{n} f_{\tilde{z}}\left(\tilde{z}_{i} \mid L\right) \\
& =(L)^{n L d} \frac{\exp \left(-L \sum_{i=1}^{n} \tilde{z}_{i}\right)}{[\Gamma(L d) \Gamma(L d, L \rho)]^{n}} \prod_{i=1}^{n} \tilde{z}_{i}^{L d-1}
\end{aligned}
$$

where $\left\{\tilde{z}_{i}\right\}_{i=1}^{n}$ is a size $n$ sample of truncated MPWF outputs. The log-likelihood function is derived as

$$
\begin{aligned}
& \log \mathcal{L}(L \mid \tilde{z})=n L d \log L-n \log \Gamma(L d, L \rho) \\
& -n \log \Gamma(L d)-n L \frac{1}{n} \sum_{i=1}^{n} \tilde{z}_{i}+n(L d-1) \frac{1}{n} \sum_{i=1}^{n} \log \left(\tilde{z}_{i}\right)
\end{aligned}
$$

where $(1 / n) \sum_{i=1}^{n} \tilde{z}_{i}$ and $(1 / n) \sum_{i=1}^{n} \log \left(\tilde{z}_{i}\right)$ are statistical means (SMs) of the original and logarithmic truncated MLC measurements. Thus, we can get the MLE estimate of the ENL

$$
\widehat{L} \subseteq \underset{L}{\arg \max }\{\log \mathfrak{L}(L \mid \tilde{z})\}
$$

which must be solved numerically. Thus both the ENL and the polarimetric covariance matrix are well estimated.

To summarize, there are two parameters that need to be estimated, the equivalent number of looks (ENL) and the mean of MPWF.

The distribution of the MPWF without the calibration of polarimetric covariance matrix is

$$
z \sim \gamma\left(L d, \frac{\mu_{T}(\rho)}{L}\right)
$$

Here we can denote $\mu=\mu_{T}(\rho)$ to simplify the notation. The PDF of $\tilde{z}$, the truncated data is

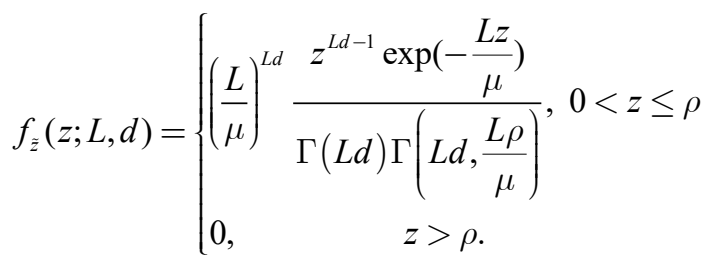

A maximum likelihood (ML) estimator can be obtained from the likelihood function

$$
\begin{aligned}
& \log \mathcal{L}(\mu, L \mid \tilde{z})=n L d \log \frac{L}{\mu}-n \log \Gamma\left(L d, \frac{L \rho}{\mu}\right)-n \log \Gamma(L d) \\
& -\frac{n L}{\mu} \frac{1}{n} \sum_{i=1}^{n} \tilde{z}_{i}+n(L d-1) \frac{1}{n} \sum_{i=1}^{n} \log \left(\tilde{z}_{i}\right)
\end{aligned}
$$

We can solve the maximization numerically to obtain the best estimations of $\mu, L$.

There are several methodologies that can estimate the ENL in polarimetric data, e.g. Anfinsen et al.. Once the ENL is estimated it can be considered as an image constant.

We are therefore left with the estimation of the mean value $\mu$. If the ENL is known to us, $\partial / \partial \mu \log \mathcal{L}(\mu, L \mid \tilde{z})=0$ can be used to derive the derivative of the log-likelihood function as

$$
f(t)=\frac{t^{L d} \exp (-t)}{\Gamma(L d) \Gamma(L d, t)}-L\left(d-\frac{\tilde{z} t}{L \rho}\right)=0
$$

where $t=\frac{L \rho}{\mu}$.

We can obtain accurate estimations of ENL by the iterative censoring (IC) scheme. In this case, the truncated radial is fixed, and the pixels of the truncated data are updated with the truncated polarimetric covariance matrix until the difference between the numbers of the truncated pixels in the IC scheme is small enough.

Although the iterative detection procedure may need several cycles and require long calculation time, the IC scheme has shown robust performance in the dense target situation and can be integrated with the CFAR algorithm.

The specified false alarm rate $P_{F A}$ can be related to the CDF, parameterized with the estimated mean value, as

$$
P_{F A}=1-F_{z}(T ; L, d)=1-\Gamma\left(L d, \frac{L T}{\mu}\right)
$$

where $T$ is the detection threshold that needs to be solved:

$$
T=\frac{\mu}{L} \Gamma^{-1}\left(L d, 1-P_{F A}\right)
$$

3) Generalized model of the TS

Since Eq (13) and Eq (21) represent gamma distribution which is a special case of the generalized gamma distribution (GГD), it is possible to make this expression more robust if we use the GГD instead as

$$
\gamma(\alpha, \beta)=f(x ; \alpha, 1, \alpha \beta)
$$

where $f$ is the GГD as follows:

$$
f(z ; k, v, \sigma)=\frac{|v| k^{k}}{\sigma \Gamma(k)}\left(\frac{z}{\sigma}\right)^{k v-1} \exp \left\{-k\left(\frac{z}{\sigma}\right)^{v}\right\}
$$

$k, v, \sigma$ are the shape, power and scale parameters, respectively. $k=L d, \sigma=d \mu$.

Its cumulative density function (CDF) is

$$
F_{z}(z ; L, d)=\left\{\begin{array}{c}
\Gamma\left[k, \eta z^{v}\right], v>0 \\
1-\Gamma\left[k, \eta z^{v}\right], v<0
\end{array}\right.
$$

where $\eta=k \sigma^{-v}$. The PDF of $\tilde{z}$, the truncated data is 


$$
f_{\tilde{z}}(\tilde{z} ; k, v, \sigma)= \begin{cases}\frac{f(z ; k, v, \sigma)}{F_{z}(\rho ; L, d)}, & 0<z \leq \rho \\ 0, & z>\rho .\end{cases}
$$

Via the definition of the incomplete gamma function, we can get the $\mathrm{n}$-th moments of the truncated data after MPWF

$$
E_{\tilde{z}}\left(\tilde{z}^{n}\right)=\frac{\eta^{-\frac{n}{v}} \Gamma\left(k+\frac{n}{v}\right) \Gamma\left(k+\frac{n}{v}, \eta \rho^{v}\right)}{\Gamma(k) \Gamma\left[k, \eta \rho^{v}\right]}
$$

The ML estimator is used.

$$
\begin{aligned}
& \log \mathcal{L}(k, v, \sigma \mid \tilde{z})=n \log |v|+n k \log k+(k v-1) \sum_{i=1}^{n} \log \left(\tilde{z}_{i}\right) \\
& -n k v \log \sigma-\frac{k}{\sigma^{v}} \sum_{i=1}^{n} \tilde{z}_{i}^{v}-n \log \Gamma(k)-n \log \Gamma\left(k, \eta \rho^{v}\right)
\end{aligned}
$$

By differentiating the log-likelihood function with respect to $k, v, \sigma$ and setting them to zero, we obtain the following three equations (34-36)

$$
\begin{aligned}
& \frac{\partial \log \mathcal{L}}{\partial k}=n+2 n \log \eta+n v \log \rho-\frac{n K}{\Gamma(k) \Gamma\left(k, \eta \rho^{v}\right)} \\
& -\frac{n}{\sigma^{v}}\left(\frac{1}{n} \sum_{i=1}^{n} \tilde{z}_{i}^{v}\right)+n v\left(\frac{1}{n} \sum_{i=1}^{n} \log \tilde{z}_{i}\right)=0 \\
& \frac{\partial \log \mathcal{L}}{\partial v}=n \frac{1}{v}+k \sum_{i=1}^{n} \log \left(\tilde{z}_{i}\right)-n k \log \sigma \\
& -n \frac{\left(\eta \rho^{v}\right)^{k} \exp \left(-\eta \rho^{v}\right) \log \rho}{\Gamma(k) \Gamma\left(k, \eta \rho^{v}\right)}-n \frac{k}{\sigma^{v}}\left(\frac{1}{n} \sum_{i=1}^{n} \tilde{z}_{i}^{v} \log \left(\tilde{z}_{i}\right)\right)=0
\end{aligned}
$$

$$
\frac{\partial \log \mathcal{L}}{\partial \sigma}=-n \frac{k v}{\sigma}+\frac{k v}{\sigma^{v+1}} \sum_{i=1}^{n} \tilde{z}_{i}^{v}+n \frac{\left(\eta \rho^{v}\right)^{k} \exp \left(-\eta \rho^{v}\right) v}{\sigma \Gamma(k) \Gamma\left(k, \eta \rho^{v}\right)}=0
$$

where $K=\Gamma(k) \psi(k)-\eta \rho^{v} T\left(3, k, \eta \rho^{v}\right), \eta=k \sigma^{-v}$, and in [49]:

$$
T(m, s, z)=G_{m-1, m}^{m, 0}\left(\begin{array}{c}
0,0, \ldots, 0 \\
s-1,-1, \ldots-1
\end{array}\right)
$$

MeijerG function is defined as

$$
\begin{aligned}
& G_{p, q}^{m, n}\left[z \mid \begin{array}{l}
a_{1}, \cdots, a_{p} \\
b_{1}, \cdots, b_{q}
\end{array}\right]=\frac{1}{2 \pi i} \\
& \times \int_{L} \frac{\prod_{j=1}^{m} \Gamma\left(b_{j}-s\right) \prod_{j=1}^{n} \Gamma\left(1-a_{j}+s\right)}{\prod_{j=m+1}^{q} \Gamma\left(1-b_{j}+s\right) \prod_{j=n+1}^{p} \Gamma\left(a_{j}-s\right)} z^{s} d s
\end{aligned}
$$

The PFA/PD can be rewritten as

$$
P_{F A}=1-F_{z}(z ; L, d)=\left\{\begin{array}{c}
1-\Gamma\left[k, \eta T^{v}\right], v>0 \\
\Gamma\left[k, \eta T^{v}\right], v<0
\end{array}\right.
$$

The threshold turns out to be

$$
T=\left\{\begin{array}{l}
\left(\frac{1}{\eta} \Gamma^{-1}\left[k, 1-P_{F A}\right]\right)^{1 / v} \\
\left(\frac{1}{\eta} \Gamma^{-1}\left[k, P_{F A}\right]\right)^{1 / v}
\end{array}\right.
$$

where $\eta=k \sigma^{-v}$. It can also be seen that the calculation of the threshold is also easier to obtain compared with the gamma distribution.

In the following we will use both the truncated gamma distribution and the truncated GГD to fit simulated and real PolSAR data.

\section{Performance of Parameter Estimation}

This section presents empirical results that a) show the correctness of our derived distributions after truncation; b) evaluates the performance of ENL estimator for truncated data, and compare with the conventional ML estimator for uncontaminated data.

Firstly, we evaluate the correctness of the distribution of the truncated data. Monte Carlo simulations are used to produce simulated SAR data. We used simulation to control exactly the statistics of clutter and contaminating targets. We generated the Wishart distribution covariance matrices and the textual variables. The Wishart part of the synthetic data set consists of $N=1,000,000$ covariance matrix samples drawn from a complex, circular, and zero-mean Wishart distribution. The distribution was colored by a matrix that is computed by averaging a homogeneous region of see in real SAR data. The number of looks in the simulation was set to $L=10$. From $N=1,000,000$ samples, we drew $\left.M=\left[\begin{array}{lll}1000 & 10000 & 100000\end{array}\right]\right)$ bootstrap samples to evaluate the correctness of the truncated distribution, which are presented in Fig 1 for Eq (15). It can be seen that the theoretical PDF is quite in accordance with the simulated data, especially when the sample size is large enough. We can get the same results of the other expressions of the truncated PDFs in Eq (23) and Eq (31).

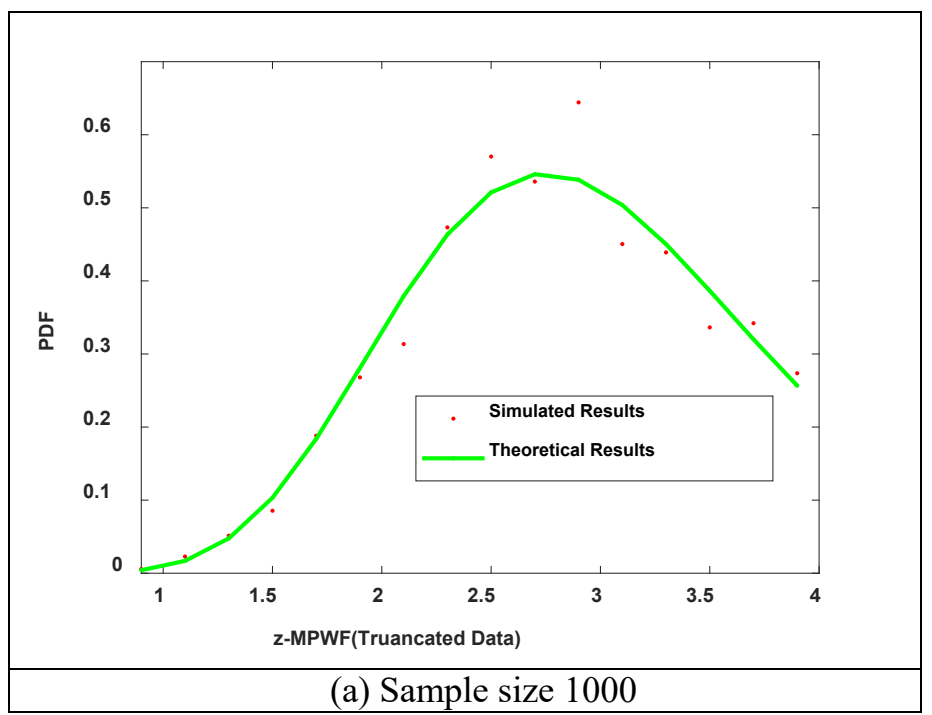




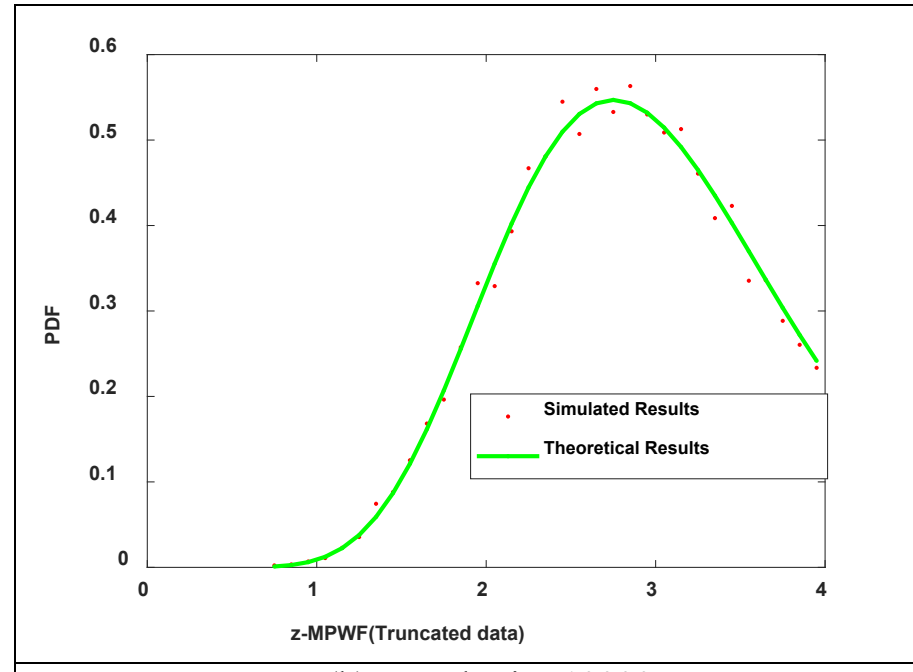

(b) Sample size 10000

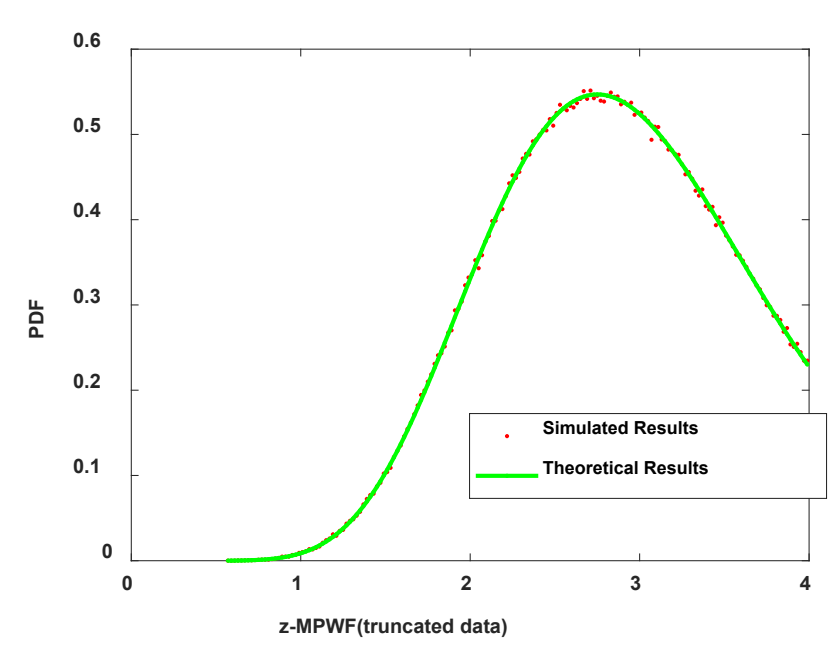

(c) Sample size 100000

Fig 1 The validation of the truncated statistics

Secondly we assess the performance of different ENL estimators by the mean square error (MSE), which is defined as

$$
\begin{aligned}
& \operatorname{Bias}(\hat{\beta})=|\mathrm{E}(\hat{\beta})-\beta| / \beta \\
& \operatorname{Var}(\hat{\beta})=\mathrm{E}\left\{[\mathrm{E}(\hat{\beta})-\hat{\beta}]^{2}\right\} / \beta^{2} \\
& \operatorname{MSE}(\hat{\beta})=\mathrm{E}\left[(\hat{\beta}-\beta)^{2}\right] / \beta^{2}=\operatorname{Bias}(\hat{\beta})^{2}+\operatorname{Var}(\hat{\beta})
\end{aligned}
$$

where $\beta$ is the true value, $\hat{\beta}$ is the estimation of $\beta$. To describe i) the simulation of contaminated data and ii) the depth of PWF truncation, we define two parameters. The contamination ratio $R_{c}$ is defined as the proportion of contaminated data points relative to the total samples size, and the truncation ratio $R_{t}$ as the fraction of truncated samples over the total samples size. Here we set the contamination ratios as $R_{c}=0 \%$ and $R_{c}=20 \%$, respectively. And the target to clutter ratio (TCR) is defined as TCR $=\operatorname{tr}\left(\boldsymbol{\Sigma}_{T}-\boldsymbol{\Sigma}_{C}\right) / \operatorname{tr}\left(\boldsymbol{\Sigma}_{C}\right)$, where $\boldsymbol{\Sigma}_{T}$ is the polarimetric covariance matrix of target and $\boldsymbol{\Sigma}_{C}$ is the polarimetric covariance matrix of sea clutter. The contamination samples are drawn from a gamma distribution
$\mathrm{TCR}=2$. Unfortunately both the estimators based on PWF-TS should solve the complicated transcendental equations numerically. We found a group of ENL estimators based on the sub-matrix and logcumulants [50], which may be used in both the untruncated and truncated data perfectly and cost little time.

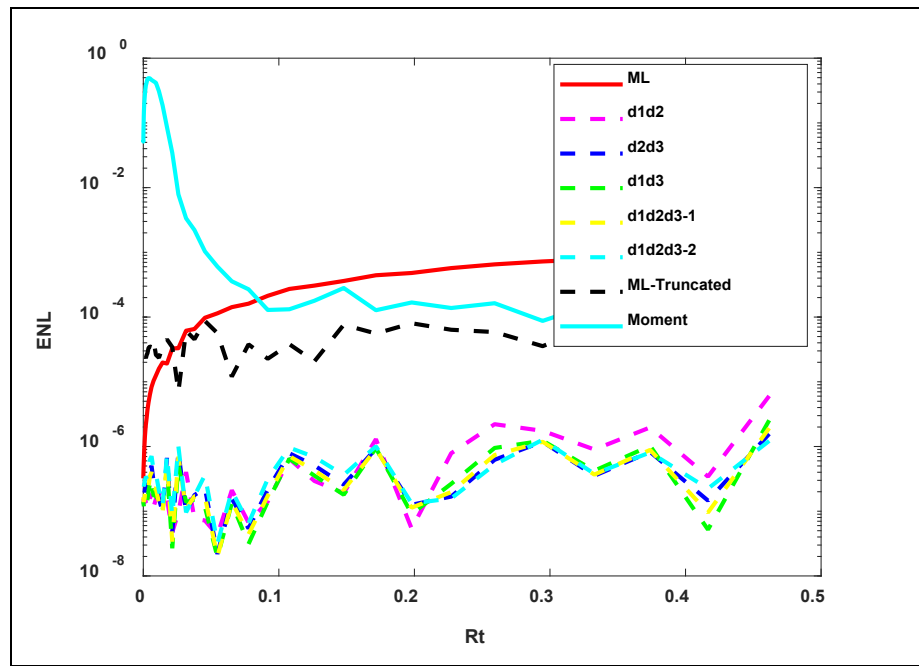

(a) Sample size 1000

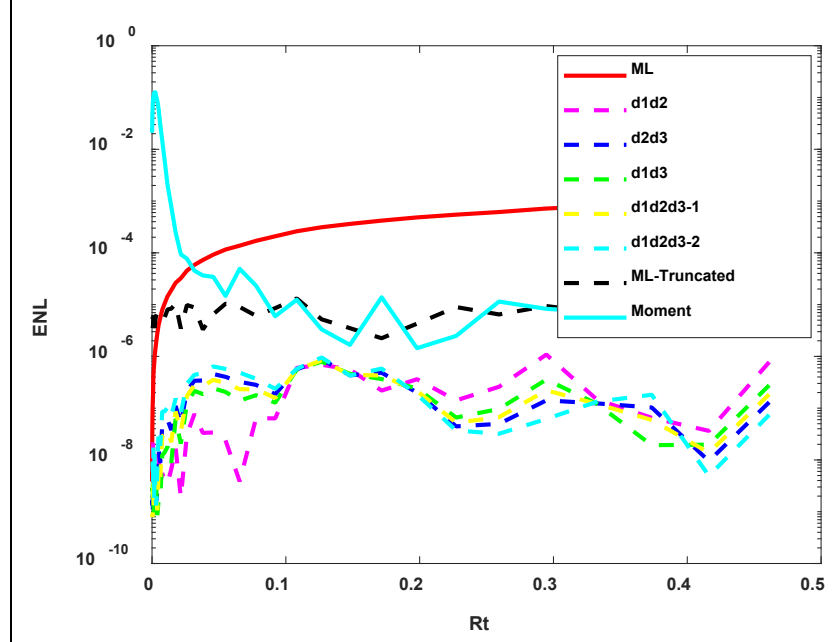

(b) Sample size 5000

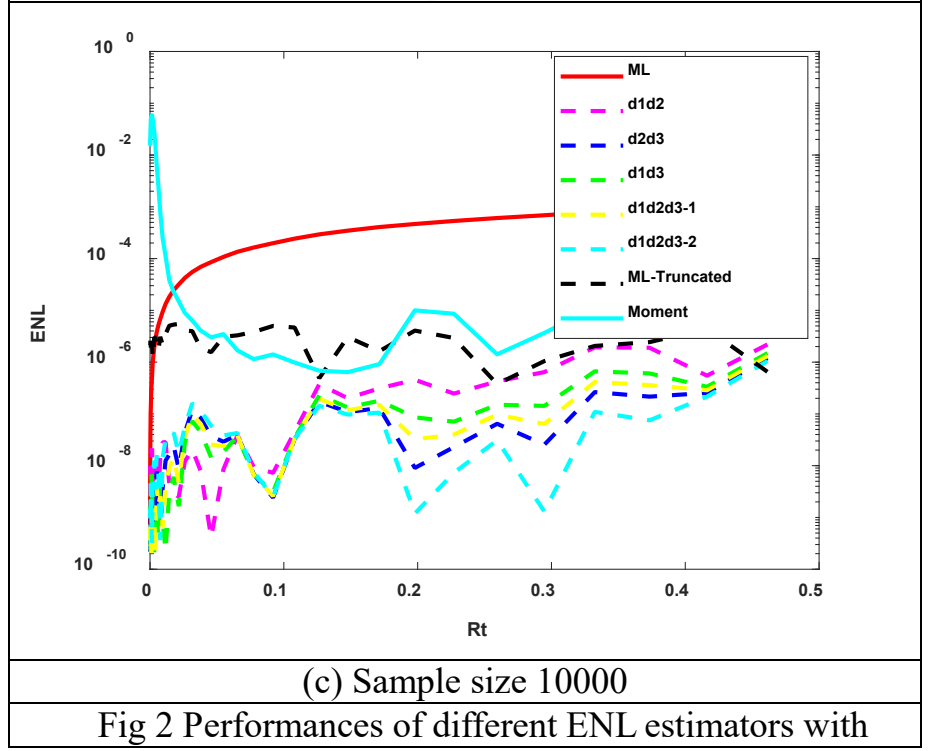


$\mathrm{Rc}=0$ and $\mathrm{TCR}=2$

The results in Fig 2 are in the case of the uncontaminated data, and the results for the contaminated data are listed in Fig 2.
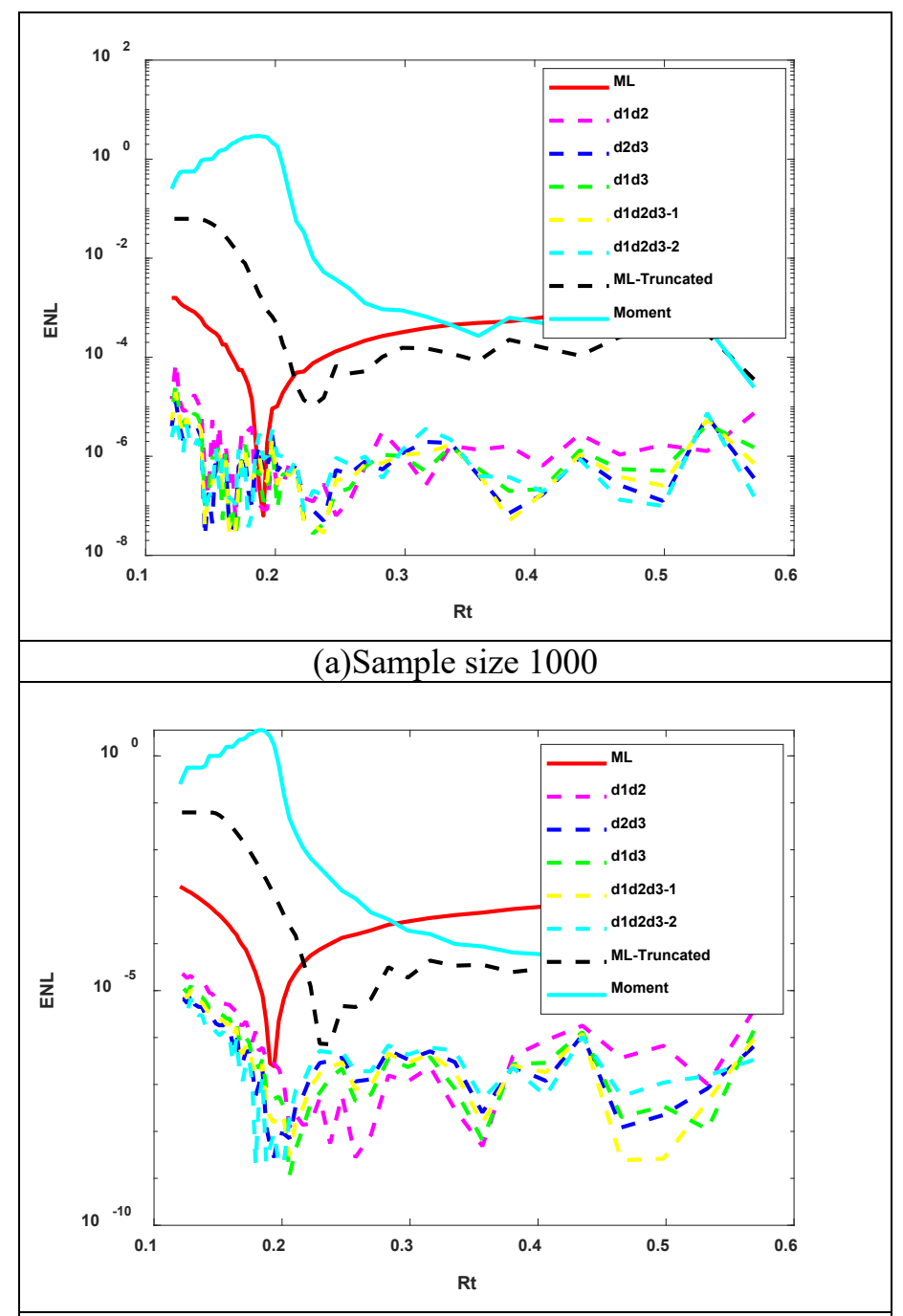

In both Fig 2 and Fig 3, d1d2, $d 2 d 3, d 1 d 3 d 1 d 2 d 3-1$ and d1d2d3-2 are all estimators based on the sub-matrices in [50]. From the results we can see the performances of the sub-matrix estimators give the best results and each of them gives similar performance. Therefore we can take one as the ENL-SM method. The ML method is proposed by S N Anfinsen, which is used for the untruncated data and can be named as ENL-SN. The ML-Truncated method is our method suitable for truncated data, which is named as ENL-ML. It can be seen when the truncated ratio almost equals the contaminated ratio, the ML and the ML-truncated methods may give a good performance.

If the ENL has been obtained, the $\mu_{T}(\rho)$ can be obtained easily via Eq (12) or the ML estimator in Eq (25). We also found the solutions via $\mathrm{Eq}(24)$ and (33) are almost the same as the above, which has also been verified by the measured data in section IV. Therefore we can choose suitable methods for parameter estimations considering both the accuracy and the efficiency.

\section{Assessment of the PWF-TS-CFAR Detector}

In this section, we use two CFAR indexes to assess the performance of the PWF-TS-CFAR detector:1) the false alarm rate maintenance and 2) the Receiver Operating Characteristic (ROC). We calculate these indexes from the simulated gamma distributed clutter. The proposed PWF-TS-CFAR detector is compared with the conventional TS-CFAR detectors based on intensity only.

1) False Alarm Rate Maintenance: The observed false alarm rate is defined as

$$
P_{f a}=\frac{n_{f a}}{n}
$$

where $n_{f a}$ is the number of false alarms and $n$ is the total number of samples. The specified false alarm rate is denoted as $P_{F A}$. A constant $P_{f a}$ can be approached to $P_{F A}$ in theory if the statistical model of clutter satisfies the assumed model and the corresponding parameter estimates are accurate. Therefore we define the False Alarm Rate Maintenance

$$
C_{L}=10 \log \left(\frac{P_{f a}}{P_{F A}}\right)
$$

This is a fundamental parameter to assess the CFAR maintenance.

2) ROC: The detection rate is measured as

$$
P_{d}=\frac{n_{d}}{n_{t}}
$$

where $n_{d}$ is the number of the detected targets and $n_{t}$ is the total number of targets. $P_{d}$ is usually evaluated at different values of $P_{F A}$ to assess detection performance, where $P_{F A}$ is chosen that produces a $P_{f a}$ that meets the practical requirements. This forms the ROC curve and it is always used to assess different detector performance. We use Monte Carlo simulations to draw the ROC curve. Here the sample size is set 1000 , which simulates the samples in a reference window. The contamination rate is set as $R_{c}=20 \%$, and the truncation ratio $R_{t}=20 \%$. The total number of simulations is 10000 , which can make $P_{f a}$ and $P_{d}$ almost the same as the true values. 


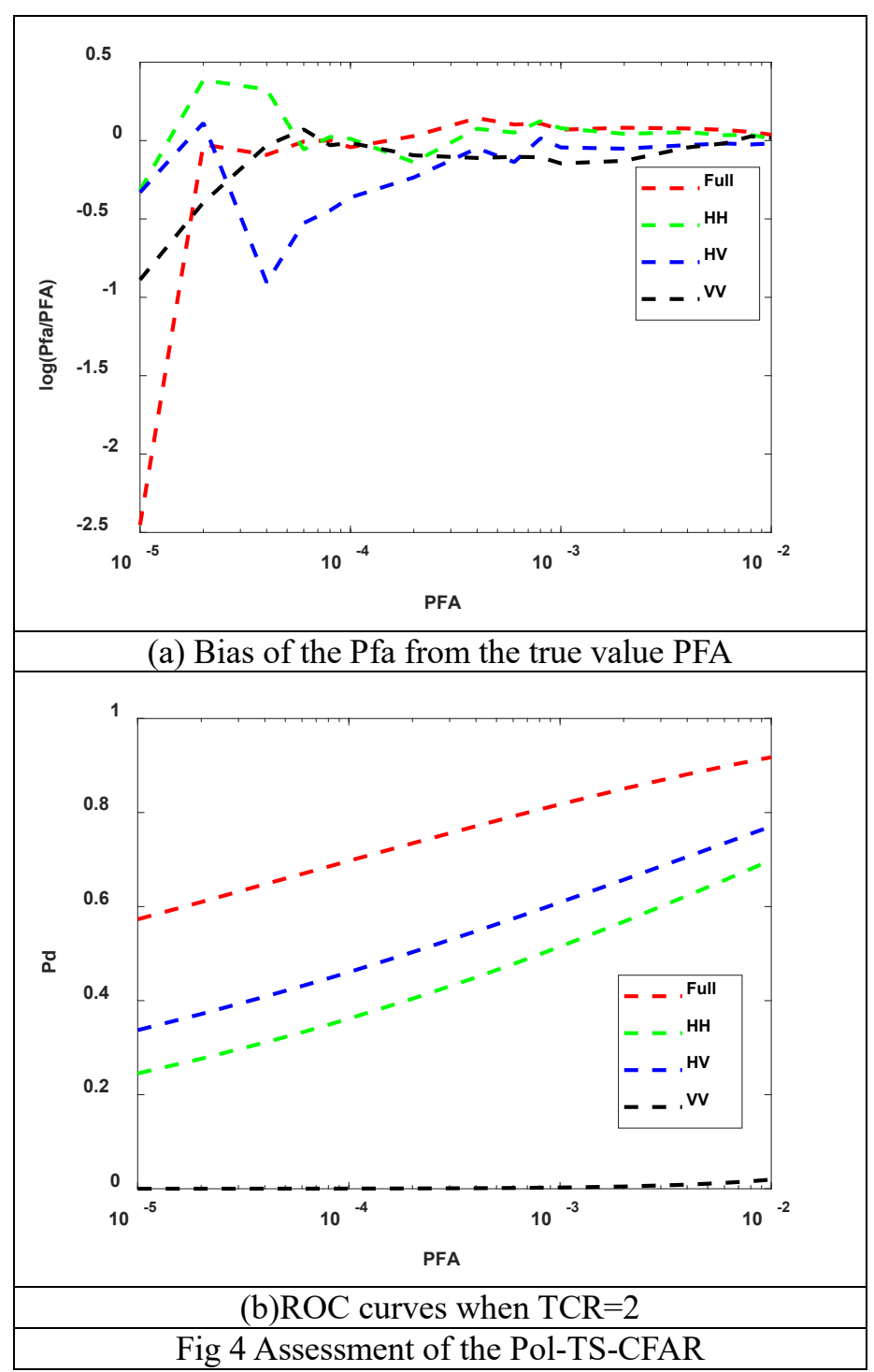

The results are presented in Fig 4. The $P_{f a}$ almost equals the PFA, which means that the truncated parameter estimation is correct and efficient. The biases of $P_{f a}$ for all truncated data in Fig 4 (a) is near to each other and gives the similar maintenance of the CFAR for values of PFA larger than 2e-5. In Fig 4 (b) it shows the full polarimetric detection gives the best performance since all polarimetric information is used. It also can be seen that the HV polarization channel is the best one for detection in all the three single polarization. The phenomenon that the VV polarization gives the worst result shows that the $\mathrm{VV}$ intensity from the targets is similar to that from the clutter, leading to the worst detection performance.

\section{Detection Performance With Real Data}

The workflow of the PWF-TS-CFAR detector proposed in this paper is presented in Fig. 5. There are three important processors, including the local detection design, the data truncation processor, and the CFAR processor. These are described in this section. The more challenging issues are the iterative censoring (IC) scheme and the adaptive setting of the truncation threshold. The workflow is indicated by the solid arrow lines. The solid parallelograms represent the main input and output. The rectangular boxes within gray background regions show the operation and procedure, and the diamond is the decision of the termination of the iterative estimation.

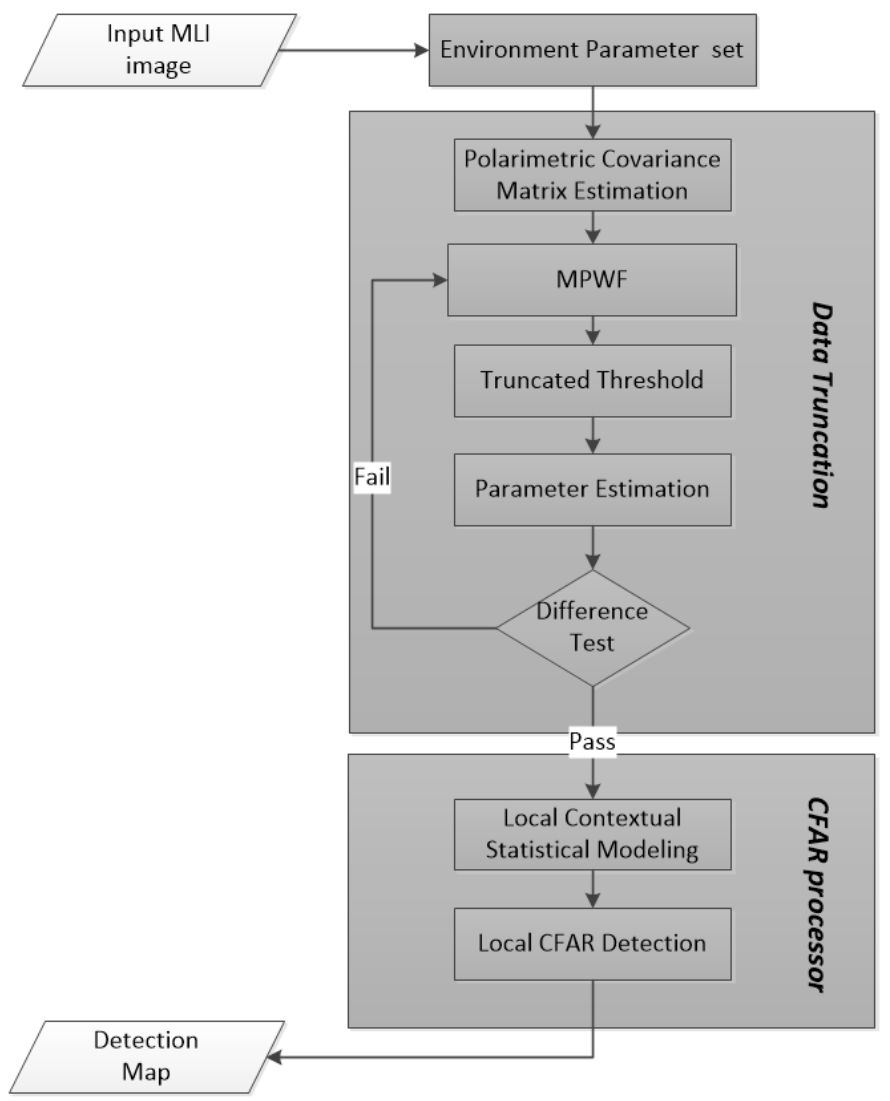

Fig 5 Workflow of the Pol-TS-CFAR detector

\section{A. Local detection Design}

To accelerate the speed of image processing, a local block detection is proposed, which consists in dividing the whole image into blocks [36][53].

As an initial step in our processing chain we choose the local block threshold algorithm in the region of interest (ROI), where the ROI is small enough to be an adequate approximation for complicated large background as well. Based on the block design and suitable estimated parameters, the local CFAR threshold for each segmented ROI can be obtained using Eq. (27) or Eq. (40). In our experiments, only one image block is presented for the sake of brevity.

\section{B. Iterative Censoring (IC) Scheme}

The iterative censoring (IC) scheme was proposed by Barboy et al to solve the parameter estimation problem in the multiple-target situation [54]. The threshold is iteratively updated based on the censored and remained reference samples. Samples get excluded from the censored reference samples when the specific samples exceed the adaptive threshold. This is repeated until there are no changes in both the threshold and the number of the reference samples [55][56]. It is no doubt that multistep adaptive detection procedures may require many cycles and cost long calculation time. In spite of this drawback, the IC scheme has shown robust performance in the multi target situation and has been integrated within CFAR algorithms. 
In our truncation processor, we first use all the pixels in the ROI to estimate the parameters and the polarimetric covariance matrix, followed by the MPWF. Then we use the fixed or the adaptive setting of truncation threshold in section $C$. Then we estimate the polarimetric covariance matrix and the other parameters by the remained pixels after truncation. Then we use the new parameters to repeat the MPWF and the following steps until the truncated number is stable.

\section{Determination of truncated threshold}

The most important thing for the truncated processor is to separate the targets from sea clutter accurately after the truncation of the MPWF. We need to set the truncated threshold suitably. Note the fact that the PDF may be bimodal. One methodology to set the truncation is by smoothing the PDF an analyzing the saddle point. In this version, we do this manually. However we will investigate automatic methodologies exploiting multimporal data for the future.

Here we evaluate the difference of PDF and fit a smoother curve. We then look for the first minimum point. Simulations are performed to validate this methodology, as shown in Fig 6. In the simulations, we first generate the sample sizes of the sea clutter (10000) and the target (2000) with the designed polarimetric covariance matrices. Then we plot the histogram of all these data after the MPWF as shown in Fig 6 (a). The first order difference of the PDF is derived, and the poly function is used to fit a curve. Generally the Polynomial order is difficult to determine, which is set 12 manually in our simulation, and therefore the truncation threshold is 6.5. The truncated result is shown in Fig 6(c). If we change the sample sizes of sea clutter and targets to be 1000 and 200 respectively, the performance is still excellent.

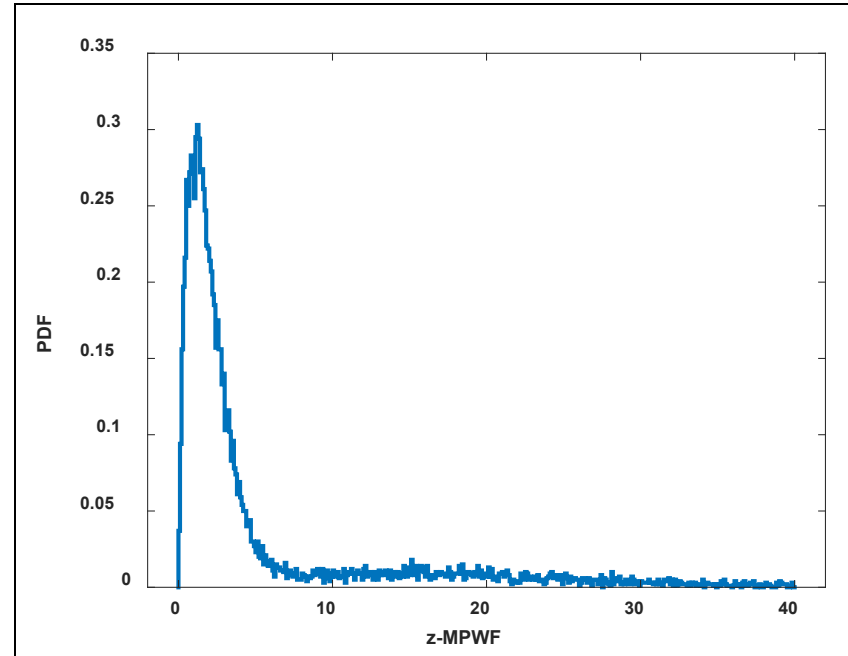

(a)the pdf of the z-MPWF
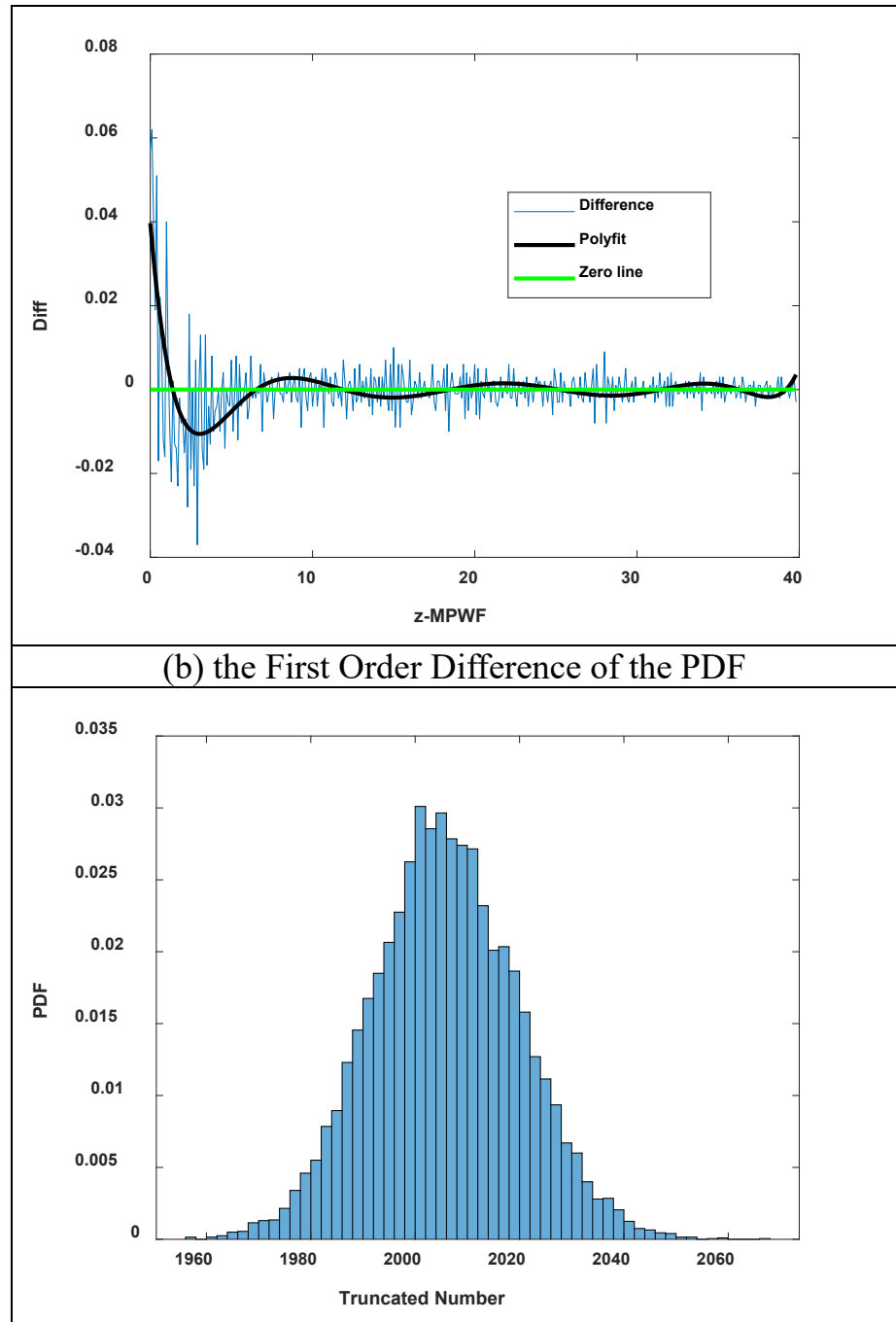

(c) the truncated result (sample size 10000)

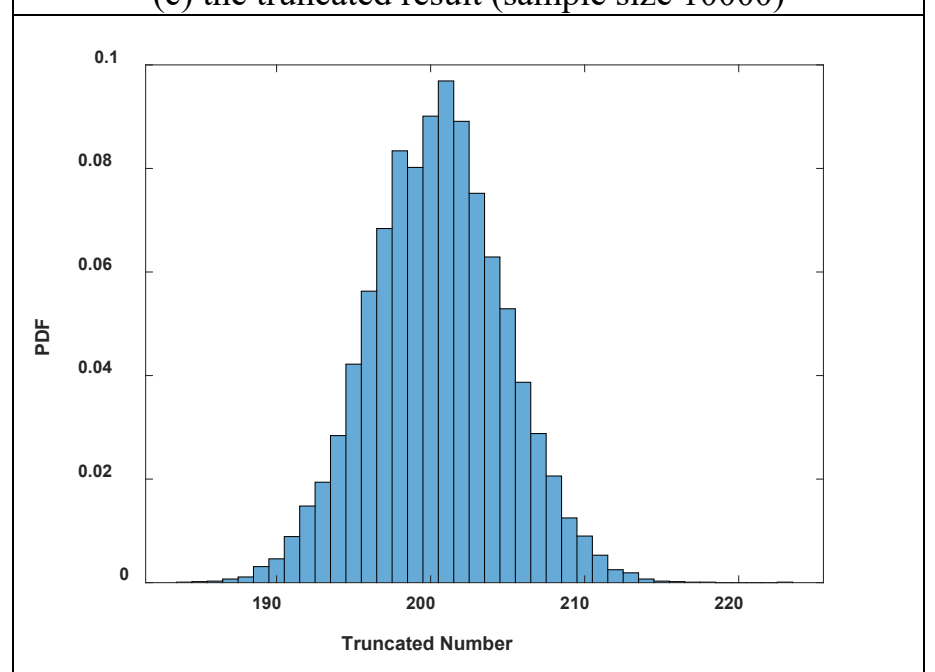

(d) the truncated result (sample size 1000)

Fig 6 Adaptive Determinant of truncated threshold

\section{Test with real data}

In this section, we test the goodness-of-fit of the statistical model for real data. Key methodologies presented in section A, $\mathrm{B}$ and $\mathrm{C}$ are applied here. Here two dataset from different satellites are used here. We first use a C-band RADARSAT-2 
polarimetric single-look complex (SLC) SAR image acquired over the Singapore Strait area [57]. The image size is 1227 (slant range) $\times 2070$ (azimuth) pixels. The nominal spatial resolution is $5.2 \mathrm{~m}$ (slant range) $\times 7.6 \mathrm{~m}$ (azimuth), and the pixel spacing is $4.7 \mathrm{~m}$ (slant range) $\times 4.8 \mathrm{~m}$ (azimuth). The incidence angle is about $47 \circ$. To perform validation, we use polarization information to assist in visually identifying small ships and remove azimuth ambiguities [58]. The final ground reference of ships marked with boxes is shown in [57], including 184. From counting the number of visible pixels of the ships, it appears that the vessels should approximately have lengths between $25 \mathrm{~m}$ and $360 \mathrm{~m}$. We choose ROI A and B randomly in Fig 7 to test our methodologies. Therefore there is no special reason for A and B are partially stacked. We use the multilook to reduce speckle, and the nominal number of looks is 4 (The multilook processing is to suppress the speckle, while it will reduce the resolution of the SAR image. Generally it is taken as 4 or 9 . Here we take it as 4 .). Since the single look detector is a special case of the multilook one, we only discuss the multilook case in our experiments.

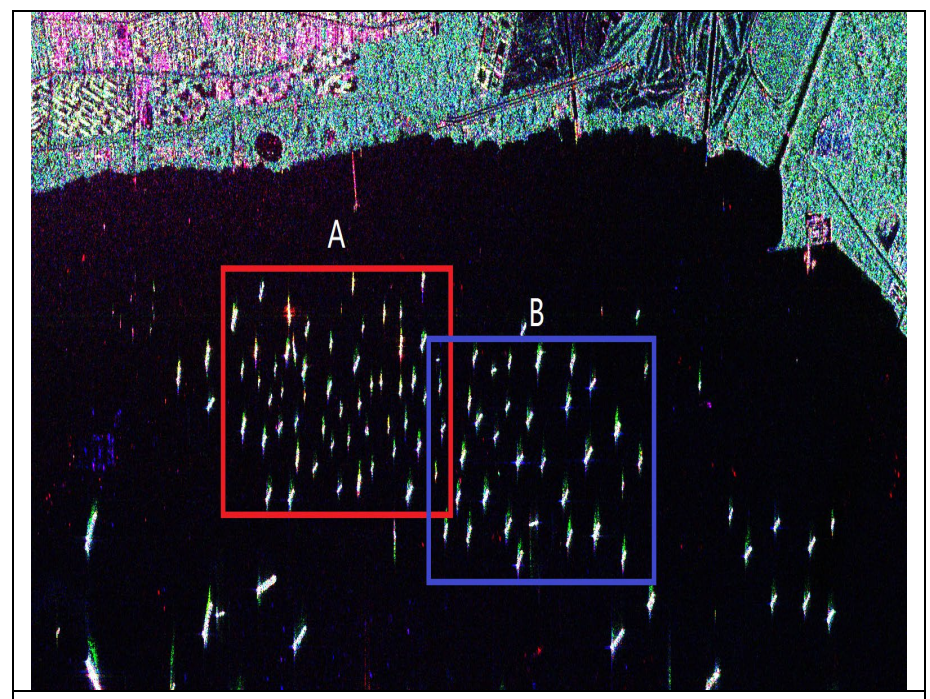

Fig 7 C-band HH polarized SLC RADARSAT-2 SAR image acquired over the Singapore Strait area.

After the multilook, the size of ROI A is $150 * 200$. The IC scheme is performed and the results of the truncation are presented in Fig 8. The truncated threshold is 6.5 , which is derived by the minimum in Fig 8(b).

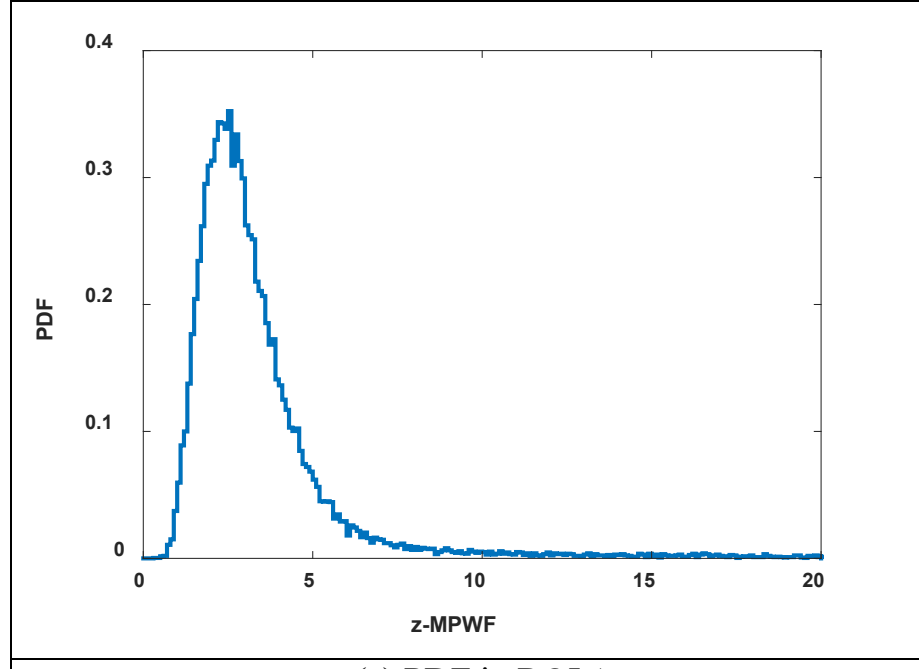

(a) PDF in ROI A

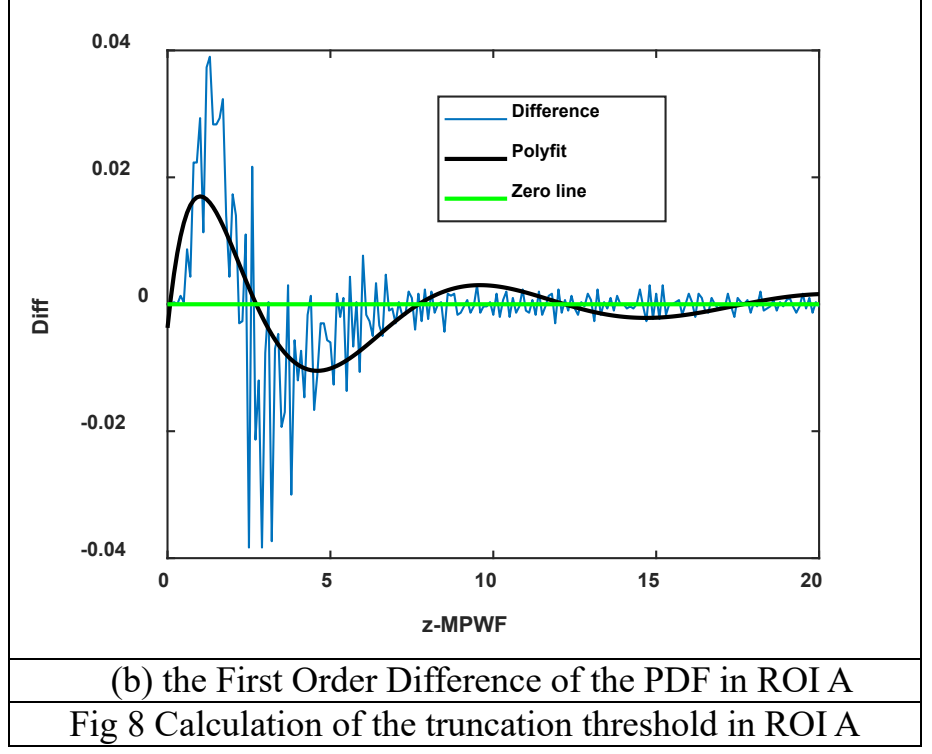

The results of the parameter estimation are listed in Table 1. The PDF of the truncated clutter is shown in Fig 9. ENL-ML is estimated using the ML criterion, and the results derived from Eq (20),(24), (25) are almost the same after the IC scheme. Therefore, we use ENL-ML to describe all the three ML estimators. ENL-SN is the estimator proposed by S N Anfinsen for the untruncated data, while processing the truncated data. Since the estimators based on the sub-matrices obtain almost the same results, we use ENL-SM to describe the sub-matrices estimators. ENL-GГD is solved by Eq (34-36). ENL-UT is the estimator proposed by $\mathrm{S} N$ Anfinsen processing the untruncated data (not doing the preprocessing of target truncation).

Table 1 The comparisons of the estimations of the ENL(A)

\begin{tabular}{|c|c|c|c|c|c|}
\hline & ENL-ML & ENL-SN & ENL-SM & ENL- GГD & ENL_UT \\
\hline $\mathrm{L}$ & 2.2390 & 3.2732 & 3.3781 & 3.3086 & 2.1561 \\
\hline $\mathrm{miu}$ & 1.0201 & 1.0067 & 1.0060 & 1.0182 & 1.0000 \\
\hline
\end{tabular}

The PDFs of different parameters estimated in table 1 are presented in Fig 9. It can be seen that the ENL- GГD gives the best performance, followed by the ENL-ML method. 


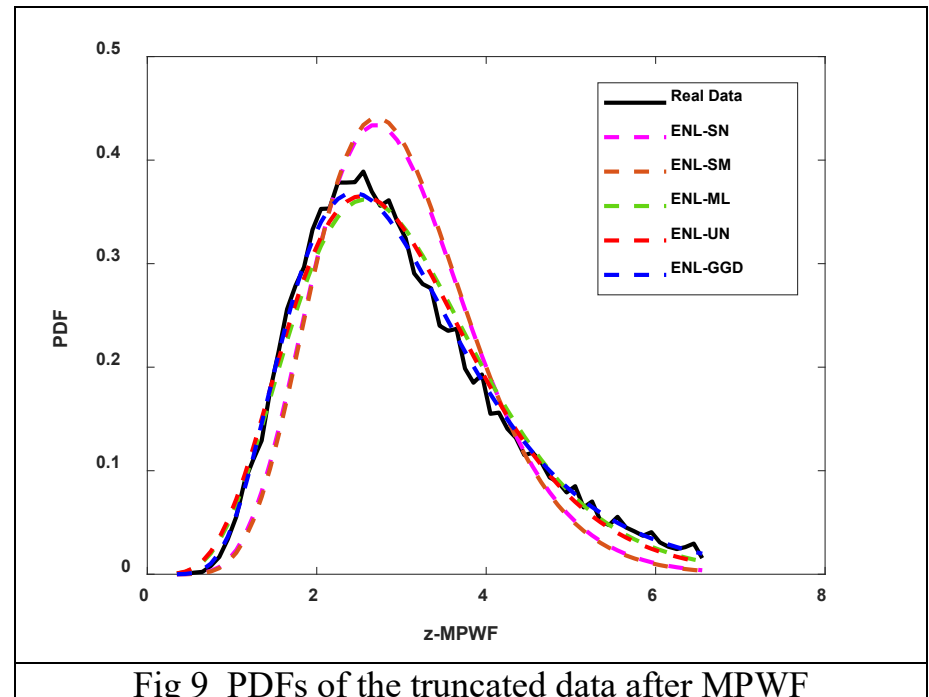

Fig 9 PDFs of the truncated data after MPWF

In Table 2, the KL distance [59] is used to measure the goodness-of-fit. The results in table 2 show the same conclusion as that from Fig 9. We can also see the ENL-ML give a good fit, whose ENL is 2.239. If we estimate the ENL by a pure sea clutter area, the ENL should be 3.2. This means the ENL estimated by the ENL-ML is to make the distribution of the clutter closest to the gamma model, while the ENL estimated here may not be the global one.

Table 2 The KL distance of the different parameters (A)

\begin{tabular}{|c|c|c|c|c|c|}
\hline & ENL_ML & ENL_SN & ENL_SM & ENL_GCD & ENL_UT \\
\hline KL & 0.0065 & 0.0413 & 0.0478 & 0.0016 & 0.0081 \\
\hline
\end{tabular}

We repeat the experiments in ROI B. The results are presented in Fig 10 ,Fig 11, Table 3 and Table 4. The size of ROI B is $200 * 150$.

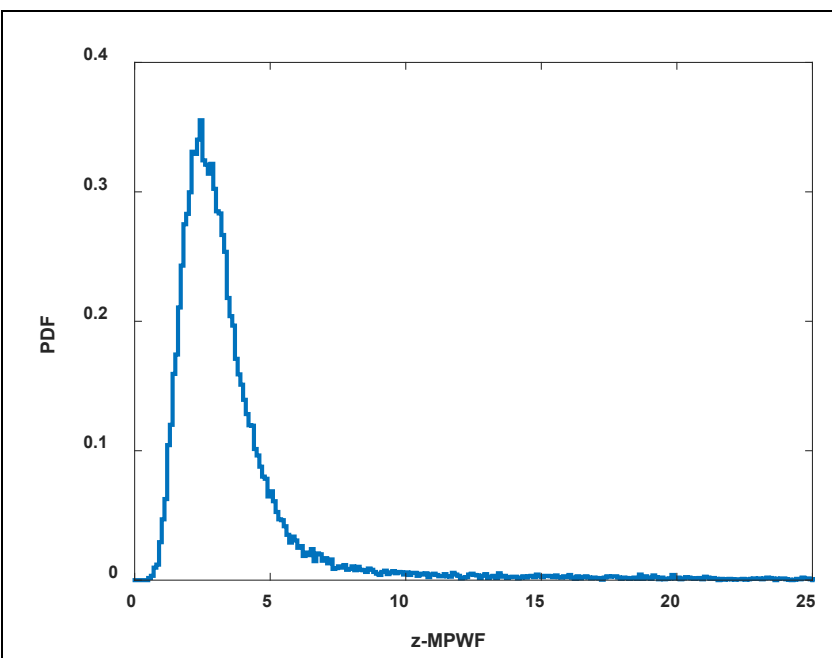

(a) PDF in ROI B

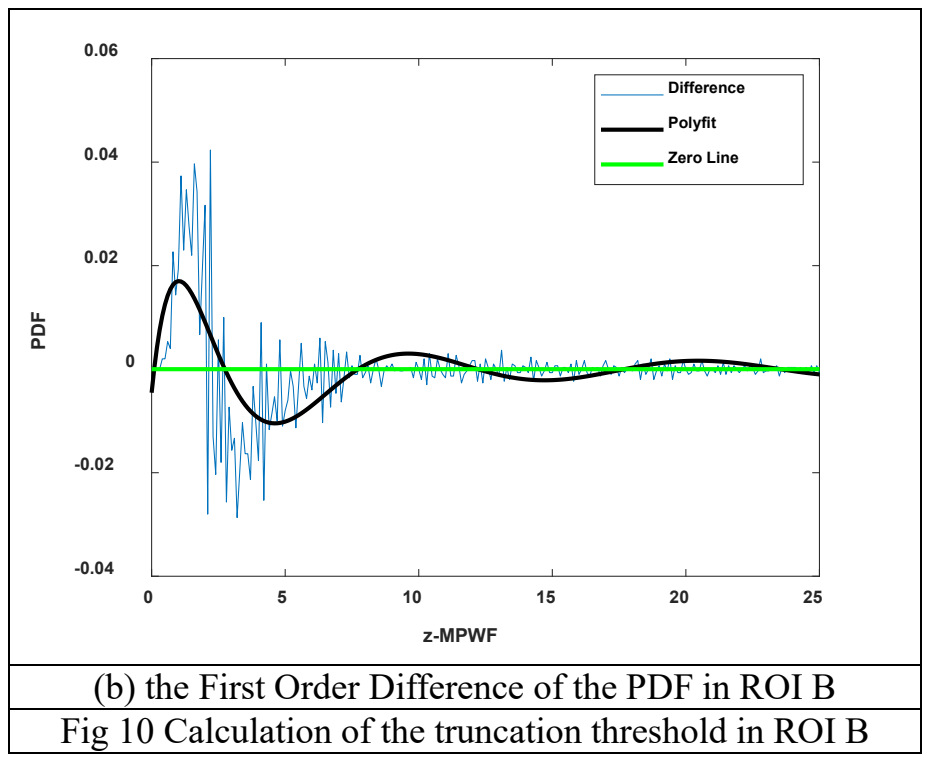

Table 3 The comparisons of the estimations of the ENL (B)

\begin{tabular}{|c|c|c|c|c|c|}
\hline & ENL_ML & ENL_SN & ENL_SM & ENL_GCD & ENL_UT \\
\hline L & 2.3085 & 3.2630 & 3.3618 & 3.3080 & 2.1546 \\
\hline miu & 1.0185 & 1.0068 & 1.0061 & 1.0160 & 1.0000 \\
\hline
\end{tabular}

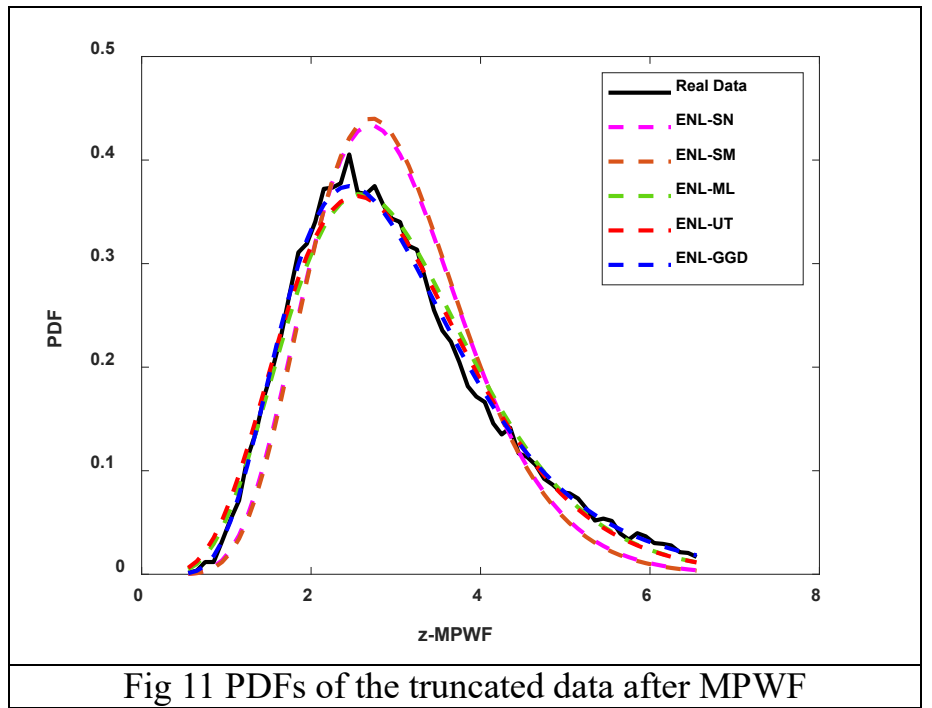

Table 4 The KL distance of the of different parameters (B)

\begin{tabular}{|c|c|c|c|c|c|}
\hline & ENL_ML & ENL_SN & ENL_SM & ENL_GГD & ENL_UT \\
\hline KL & 0.0059 & 0.0341 & 0.0397 & 0.0013 & 0.0078 \\
\hline
\end{tabular}

The conclusion in ROI B is the same as in ROI A. The results show that ENL-GГD estimator and ENL-ML estimator give better performance than the other estimators. In this dataset, we can conclude that these two estimators give better detection performance than the others we tested.

The second dataset is from the platform of GF-3, which is also works in $\mathrm{C}$ band. The scene id is 3180124 and the image mode is QPSI. The nominal number of looks is 4.The final ground truth is obtained by the same way addressed in the RadarSat-2 dataset. Five objects are obtained in the ROI C. The biggest are oil platforms, and the others are ships. The ROI C is presented in Fig 12. 


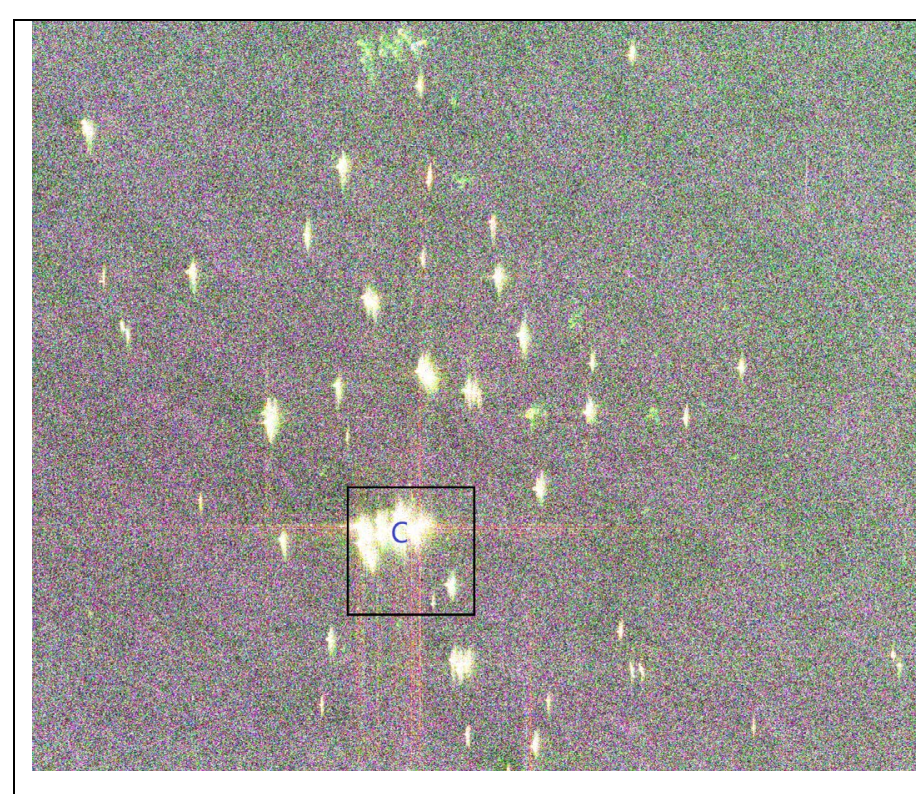

Fig 12 C-band HH polarized SLC GF-3 SAR image acquired in South China sea.

The results of the statistics and parameter estimations are listed in Fig 13-14 and Table 5-6.

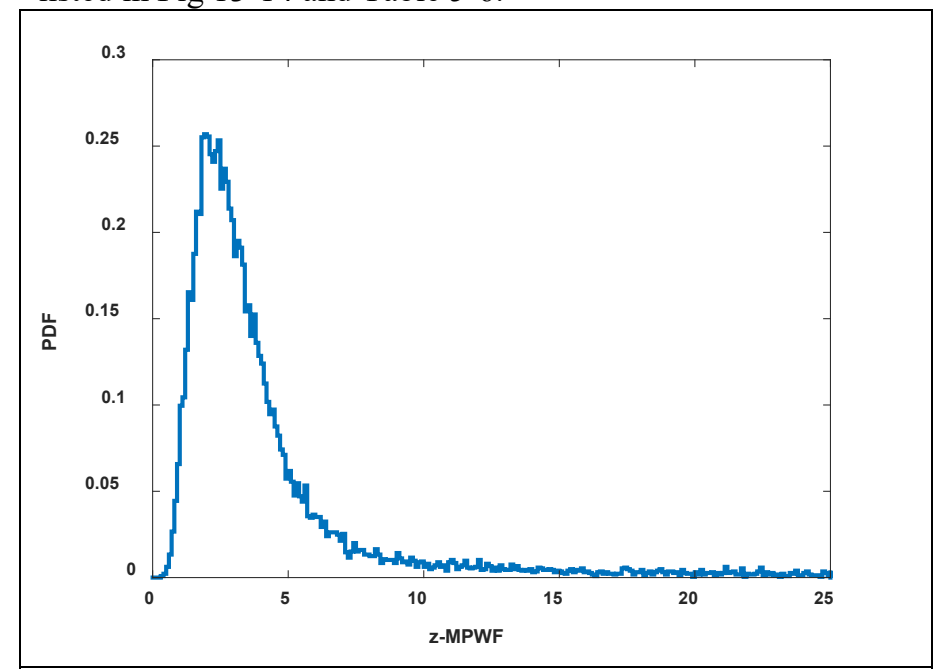

(a) PDF in ROI C

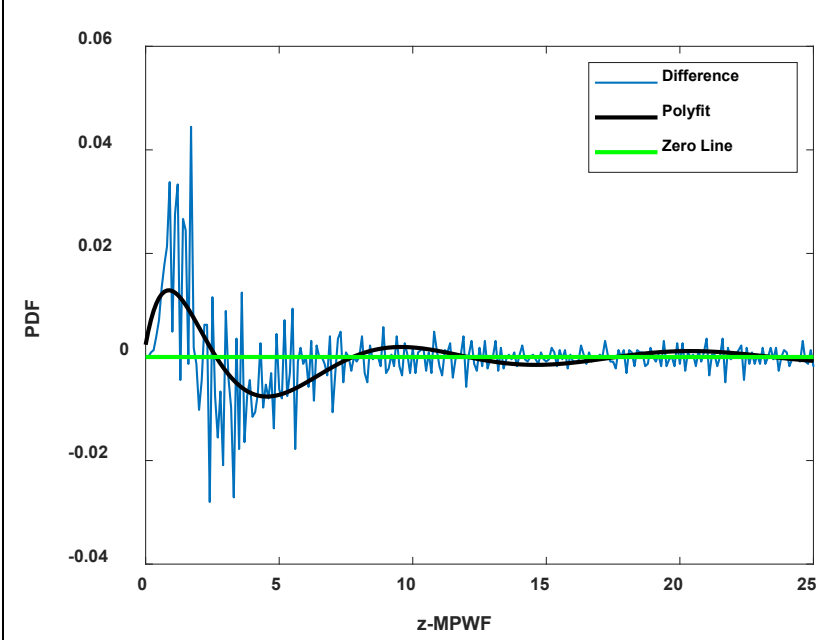

(b) the First Order Difference of the PDF in ROI C
Fig 13 Calculation of the truncation threshold in ROI C The truncation threshold is determined as 6.8 in ROI C. Table 5 The comparisons of the estimations of the ENL (B)

\begin{tabular}{|c|c|c|c|c|c|}
\hline & ENL_ML & ENL_SN & ENL_SM & ENL_GCD & ENL_UT \\
\hline L & 1.5595 & 2.7696 & 2.9024 & 2.7989 & 2.1248 \\
\hline miu & 1.0301 & 1.0038 & 1.0032 & 1.0039 & 1.0000 \\
\hline
\end{tabular}

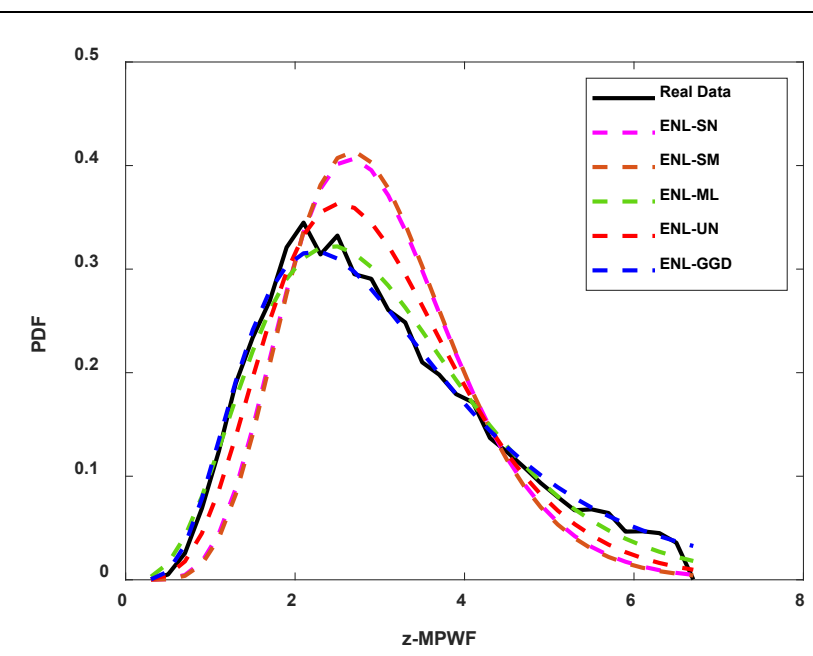

Fig 14 PDFs of the truncated data after MPWF (ROI C)

Table 6 The KL distance of the of different parameters (C)

\begin{tabular}{c|c|c|c|c|c|} 
& ENL_ML & ENL_SN & ENL_SM & ENL_GCD & ENL_UT \\
\hline KL & 0.0080 & 0.0947 & 0.1117 & 0.0017 & 0.0304 \\
\hline
\end{tabular}

The conclusion about different estimators is the same as that in ROI A and ROI B.

\section{E. Detection Performance Analysis}

To check the performance of the CFAR, we use the density-based clustering method named Density-Based Spatial Clustering of Applications with Noise (DBSCAN) [60]. We can validate that the single pixel detected in this image frame can be associated to false alarms. Please note this is not true in every dataset where the size of ships can be smaller. Based on the previous visual inspection, we set the circle radial eps $=25$ and minimum points $\mathrm{MinPt}=2$, which means 2 points in a circle with radius of $25 \mathrm{~m}$ can be seen as a ship. This applies a second layer of false alarm rejections. It should be noted that we use this clustering filter only to find points that can be associated to false alarms.

In addition, a figure of merit (FOM) is used to evaluate the detection performance [61]

$$
F o M=\frac{N_{t d}}{\left(N_{f a}+N_{g t}\right)}
$$

where $N_{t d}$ is the number of detected ships, $N_{f a}$ is the number of false ships, $N_{g t}$ is the number of real ships in the ROI. In the following figures a red rectangle means an omitted target, a yellow rectangle means a false target, and a green rectangle means one true ship.

We use the parameters estimated in Section IV.D to solve the threshold of the CFAR detection via Eq (27) and (40). And the detection performances are presented as follows. The 
MPWF- $x \times$ means the different estimators after MPWF, according to the ENL- $\times \times$ methods. The MPWF-UT means the output of MPWF without data truncation. The ground truth is presented in the Pauli-RGB image.

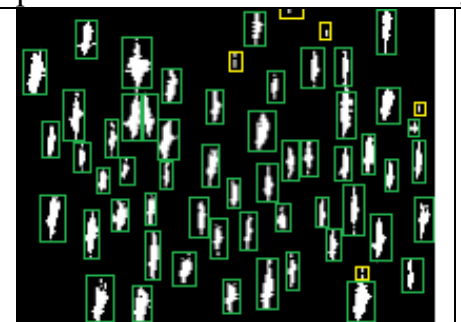

MPWF- GTD

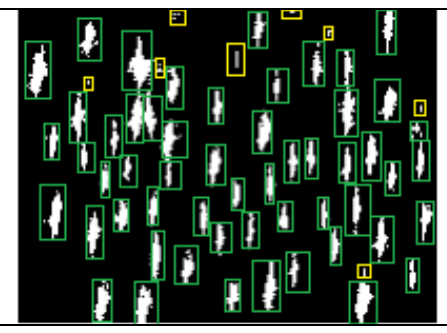
MPWF SN

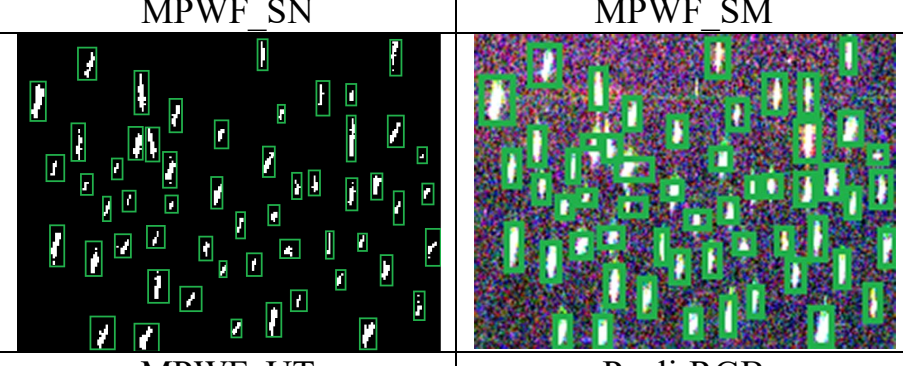
MPWF UT

Pauli-RGB

Fig 15 Detection Results of the CFAR detector based on MPWF (ROIA)

The PFA is set 0.0001 . The results are presented as follows. The Pfa and the FoM in ROI A are listed in table 5.

TABLE 7

PERFORMANCES OF ALL DETECTORS IN ROI A

\begin{tabular}{cccccc}
\hline \hline \multirow{2}{*}{ Area } & Method & $\hat{P}_{f a}$ & $N_{t d}$ & $N_{f a}$ & FoM(\%) \\
\hline \multirow{4}{*}{$\mathrm{A}$} & ENL-UT & 0.0001 & 55 & 0 & 100.00 \\
\cline { 2 - 6 } & ENL_ML & 0.0005 & 55 & 5 & 91.67 \\
\cline { 2 - 6 } & ENL_SN & 0.0014 & 55 & 8 & 87.30 \\
\cline { 2 - 6 } & ENL_SM & 0.0014 & 55 & 8 & 87.30 \\
\cline { 2 - 6 } & ENL_GCD & 0.0002 & 55 & 5 & 91.67 \\
\hline
\end{tabular}

The experiments are repeated in ROI B. The results are presented in Fig as follows.

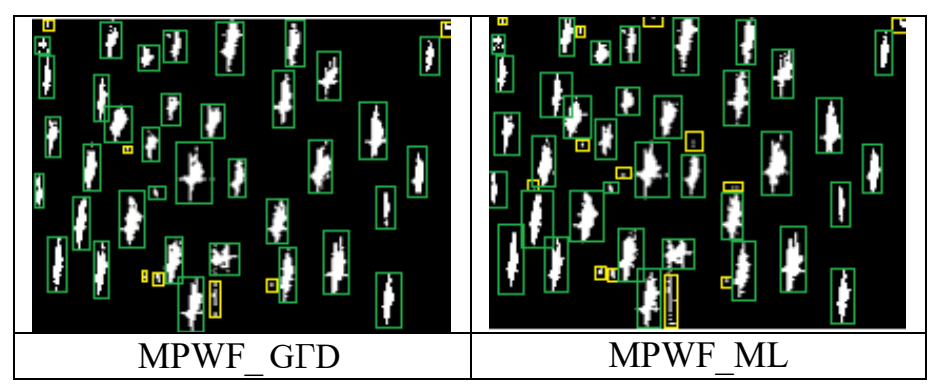

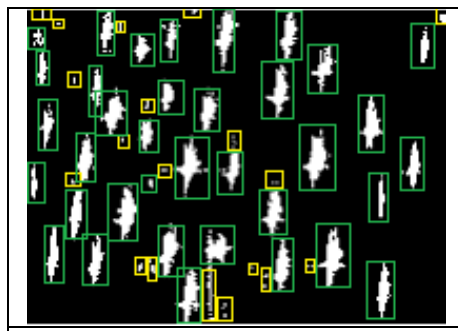

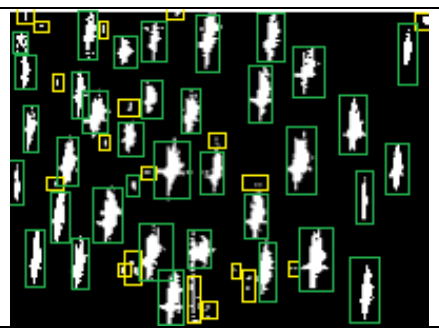

MPWF SN

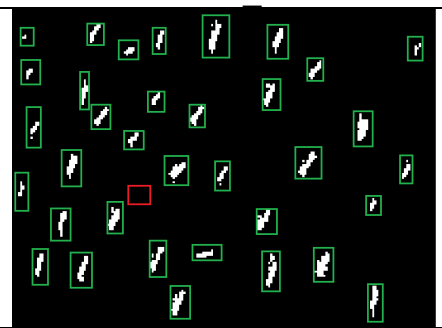

MPWF UT

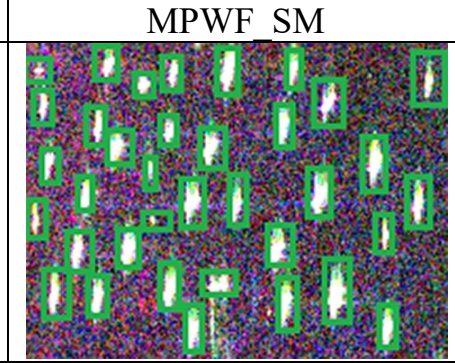

Pauli-RGB

Fig 16 Detection Results of the CFAR detector based on MPWF (ROI B)

The Pfa and the FoM in ROI B are listed in table 6.

TABLE 8

PERFORMANCES OF ALL DETECTORS IN K CASE

\begin{tabular}{cccccc}
\hline \hline Area & Method & $\hat{P}_{f a}$ & $N_{t d}$ & $N_{f a}$ & FoM $(\%)$ \\
\hline \multirow{4}{*}{ B } & ENL_UT & 0.0002 & 35 & 0 & 97.22 \\
\cline { 2 - 6 } & ENL_ML & 0.0010 & 36 & 13 & 73.47 \\
\cline { 2 - 6 } & ENL_SN & 0.0017 & 36 & 19 & 65.45 \\
\cline { 2 - 6 } & ENL_SM & 0.0017 & 36 & 19 & 65.45 \\
\cline { 2 - 6 } & ENL_GCD & 0.0002 & 36 & 7 & 83.72 \\
\hline
\end{tabular}

From the results show that the two parameters ML (ENL-ML) has the same performance compared with the three parameters GCD (ENL-GCD). However, we can expect that if the sea clutter turns to be complicated the ENL- GTD may provide better performance than the ENL-ML. Though the ENL_UT gives the best FOM, it omitted one target, and from all the figures we can see it omitted many pixels of each ship.

Moreover, in Fig. 12 and Fig. 13 some of the detected false alarms are very small compared to the real targets, and they are close to the real targets. In other words, these false alarms are phenomena generated by ship sidelobes, or ship wakes; additionally other false alarms may be generated by azimuth ambiguities.

To validate the robustness to different satellites, the GF3 data are also tested. The experiments are repeated in ROI C. The results are presented in Fig 17 and Table 9 as follows.

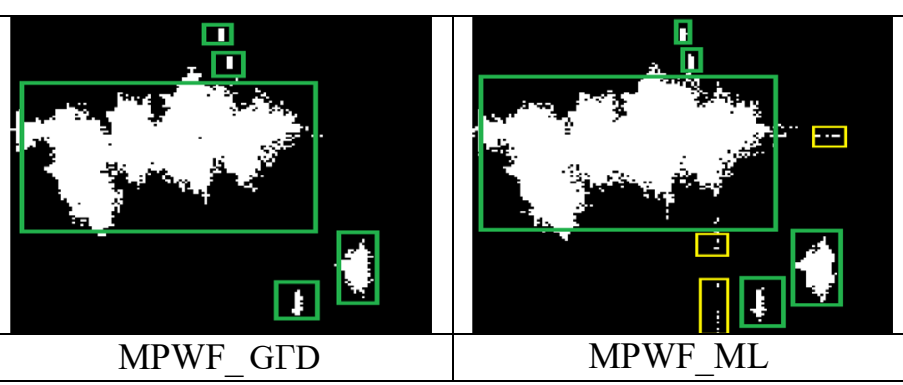




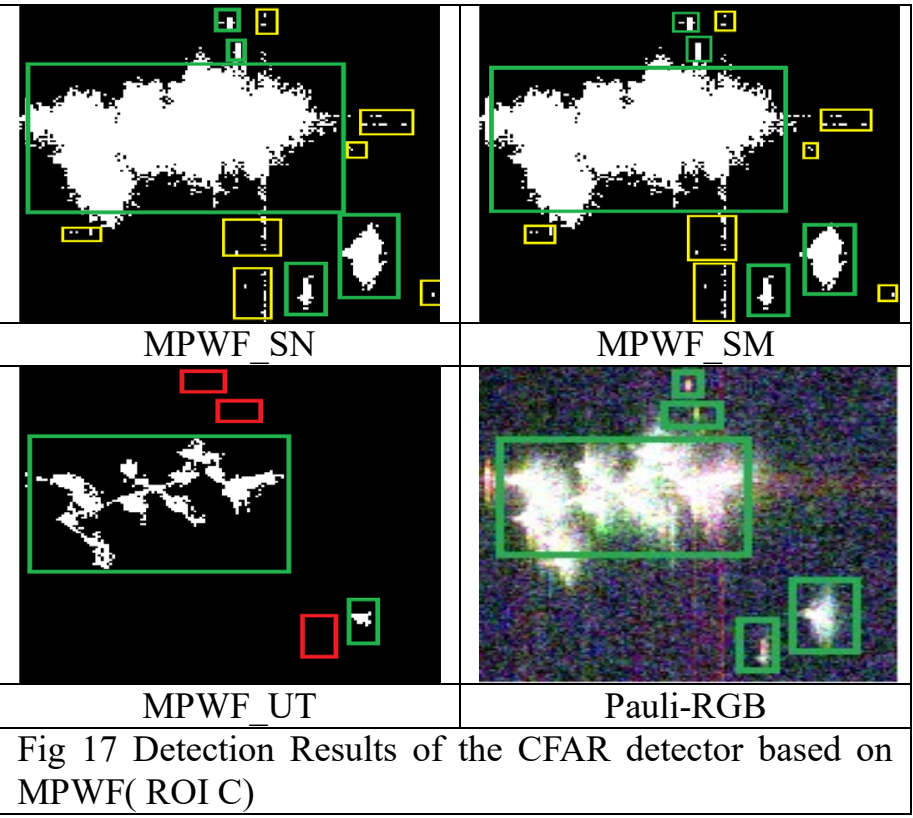

The Pfa and the FoM in ROI C are listed in table 9.

TABLE 9 PERFORMANCES OF ALL DETECTORS IN K CASE

\begin{tabular}{cccccc}
\hline \hline Area & Method & $\hat{P}_{f a}$ & $N_{t d}$ & $N_{f a}$ & $F o M(\%)$ \\
\hline \multirow{4}{*}{ C } & ENL_UT & 0.0001 & 2 & 0 & 40.00 \\
\cline { 2 - 6 } & ENL_ML & 0.0004 & 5 & 3 & 62.50 \\
\cline { 2 - 6 } & ENL_SN & 0.0014 & 5 & 7 & 41.67 \\
\cline { 2 - 6 } & ENL_SM & 0.0012 & 5 & 7 & 41.67 \\
\cline { 2 - 6 } & ENL_GSD & 0.0002 & 5 & 0 & 100.00 \\
\hline
\end{tabular}

Here we can see the MPWF- GTD gives the best performance, but the MPWF-UT with no truncation omitted all the small ships, which has a weak ability to detect small targets.

\section{CONCLUSION}

In this paper the PWF-TS-CFAR detector is proposed to improve the accuracy of estimated parameters for the clutter statistics in multiple-target situations. The PWF is a very good method for ship detection when the a priori information of targets is absent. The PWF is applied to perform the truncation of PolSAR data. The estimation of the covariance matrix after truncation is derived in appendix. PWF-TS-CFAR does not require guard window since the targets within the reference region is truncated before the clutter estimation. Therefore, it can also be used by a segmentation method [36] where there are no guard cells.

Since the degree of polarization, which has been reviewed in the introduction, provides very important information that is not covered by conventional covariance matrix optimization tools such as the PWF detector, it would be good to compare the results we have obtained in this study with a new investigation that used the minimum degree of polarization in future.

\section{APPENDIX}

In the appendix, we will derive the relation between the covariance matrix of the truncated data and that of the untruncated data following Tallis' method in [35]. From Tallis' paper, it turns out that [35]

$$
\mathfrak{S}(\rho ; \boldsymbol{\Sigma})=\frac{\boldsymbol{\Sigma}}{\mu_{T}(\rho)}, \mu_{T}(\rho) \equiv \frac{F_{d}(\rho)}{F_{d+2}(\rho)}
$$

with $F_{v}(\bullet)$ denoting the $\mathrm{CDF}$ of a $\chi^{2}$ variable with $d$ degrees of freedom. It is trivial to invert (18) so as to express $\Sigma$ as a function of $\mathfrak{S}$. This is because $c_{T}(\rho)$ is a scalar damping factor independent of $\boldsymbol{\Sigma}$.This result is only suitable for real Gaussian distributed vectors or covariance matrices.

The zero mean $d$-variate complex Gaussian under elliptical truncation is

$$
p(\mathbf{x})=\frac{1}{\pi^{d}|\mathbf{\Sigma}|} \exp \left(-\mathbf{x}^{H} \mathbf{\Sigma}^{-1} \mathbf{x}\right)
$$

According to the methods in [35][48], and the relations between the $\chi^{2}$ cumulative distribution $F_{v}(\bullet)$ and the incomplete gamma function $\Gamma(a, b)[39]$, we can extended the real number results to the complex case

$$
\boldsymbol{\Sigma}=\mu_{T}(\rho) \mathfrak{S}(\rho ; \boldsymbol{\Sigma}), \mu_{T}(\rho) \equiv \frac{\Gamma(d, \rho)}{\Gamma(d+1, \rho)}
$$

with $\Gamma(a, b)=\frac{1}{\Gamma(a)} \int_{0}^{b} y^{a-1} e^{-y} d y$.

The joint PDF of $L$ independent samples based on (A.2) should be

$$
p\left(\mathbf{x}_{1}, \mathbf{x}_{2}, \ldots, \mathbf{x}_{L}\right)=\frac{1}{\pi^{L d}|\mathbf{\Sigma}|^{L}} \exp \left(-\sum_{i=1}^{L} \mathbf{x}_{i}^{H} \mathbf{\Sigma}^{-1} \mathbf{x}_{i}\right)
$$

where $\boldsymbol{\Sigma}$ is positive semi- definite Hermitian, $\mathrm{H}$ is the conjunction transpose operator and define a set $\mathfrak{D}$ as the limitation space as follows

$$
\mathfrak{D}=\left\{\mathbf{x} \mid 0 \leq \sum_{i=1}^{L} \mathbf{x}_{i}^{H} \boldsymbol{\Sigma}^{-1} \mathbf{x}_{i}=L \operatorname{tr}\left(\boldsymbol{\Sigma}^{-1} \mathbf{C}\right) \leq L \rho\right\}
$$

where $\operatorname{tr}()$ is the trace operator, $\rho$ is the truncation radius and the 5 multilook polarimetric covariance matrix is $\mathbf{C}=\frac{1}{L} \sum_{i=1}^{L} \mathbf{x}_{i} \mathbf{x}_{i}^{\mathrm{H}}$.

(A.4) can be seen as a general form of (A.2), and the only differences are the dimension of the vector and the polarimetric covariance matrix. We can denote $\mathbf{y}=\left(\mathbf{x}_{1}, \mathbf{x}_{2}, \ldots, \mathbf{x}_{L}\right)$, and then Eq (A.2) turns to be

$$
p(\mathbf{y})=\frac{1}{\pi^{L d}|\mathbf{\Sigma}|^{L}} \exp \left(-\mathbf{x}_{i}^{H} \boldsymbol{\Xi}^{-1} \mathbf{x}_{i}\right)
$$

where $\boldsymbol{\Xi}=\left[\begin{array}{cccc}\mathbf{\Sigma} & 0 & \ldots & 0 \\ 0 & \boldsymbol{\Sigma} & \ddots & 0 \\ \vdots & \ddots & \ddots & \vdots \\ 0 & 0 & \ldots & \boldsymbol{\Sigma}\end{array}\right]$ is the $L d$ dimension covariance

matrix.

Obviously, (A.6) is the same form as (A.2). The difference between them is the dimension. Therefore we get the second moments of the truncated matrix $\Theta(\rho ; \boldsymbol{\Sigma})$ as 


$$
\boldsymbol{\Xi}=\mu_{T}(\rho) \Theta(\rho ; \boldsymbol{\Sigma}), \mu_{T}(\rho) \equiv \frac{\Gamma(L d, \rho)}{\Gamma(L d+1, \rho)}
$$

Observing the form of the $L d$ dimension polarimetric covariance matrix is a block diagonal matrix, we can get the second moments of the truncated matrix $\mathfrak{S}(\rho ; \boldsymbol{\Sigma})$ as

$$
\boldsymbol{\Sigma}=\mu_{T}(\rho) \mathfrak{S}(\rho ; \boldsymbol{\Sigma}), \mu_{T}(\rho) \equiv \frac{\Gamma(L d, \rho)}{\Gamma(L d+1, \rho)}
$$

\section{REFERENCE}

[1] A. Marino, S. R. Cloude, and I. H. Woodhouse, "Detecting depolarized targets using a new geometrical perturbation filter," IEEE Transactions on Geoscience and Remote Sensing., vol. 50, no. 10, pp. 3787-3799, Oct. 2012.

[2] L. M. Novak, M. B. Sechtin and M. J. Cardullo, "Studies of target detection algorithms that use polarimetric radar data," IEEE Transactions on Aerospace and Electronic Systems, vol. 25, no. 2, pp. 150-165, March 1989.

[3] S. R. Cloude and E. Pottier, "A review of target decomposition theorems in radar polarimetry," IEEE Trans. Geosci. Remote Sens., vol. 34, no. 2, pp. 498-518, Mar. 1996.

[4] R. Ringrose and N. Harris, "Ship detection using polarimetric SAR data," in Proc. Eur. Space Agency (ESA-SP), vol. 450, Mar. 2000, pp. 687.

[5] J. Chen, Y. Chen, and J. Yang, "Ship detection using polarization crossentropy," IEEE Geosci. Remote Sens. Lett., vol. 6, no. 4, pp. 723-727, Oct. 2009.

[6] T. Zhang, J. Ji, X. Li, W. Yu and H. Xiong, "Ship Detection From PolSAR Imagery Using the Complete Polarimetric Covariance Difference Matrix," in IEEE Transactions on Geoscience and Remote Sensing, vol. 57, no. 5, pp. 2824-2839, May 2019.

[7] Z. Xu, B. Tang and S. Cheng, "Faint Ship Wake Detection in PolSAR Images," IEEE Geoscience and Remote Sensing Letters, vol. 15, no. 7, pp. 1055-1059, July 2018.

[8] F. Biondi, "Low-Rank Plus Sparse Decomposition and Localized Radon Transform for Ship-Wake Detection in Synthetic Aperture Radar Images," IEEE Geoscience and Remote Sensing Letters., vol. 15, no. 1, pp. 117-121, Jan. 2018.

[9] F. Biondi, "A Polarimetric Extension of Low-Rank Plus Sparse Decomposition and Radon Transform for Ship Wake Detection in Synthetic Aperture Radar Images," IEEE Geoscience and Remote Sensing Letters., vol. 16, no. 1, pp. 75-79, Jan. 2019.

[10] A. De Maio, D. Orlando, and L. Pallotta, et al., "A Multifamily GLRT for Oil Spill Detection," IEEE Transactions on Geoscience and Remote Sensing., vol. 55, no. 1, pp. 63-79, Jan. 2017.

[11] A. Marino, "A notch filter for ship detection with polarimetric SAR data," IEEE Journal of Selected Topics in Applied Earth Observation and Remote Sensing, vol. 6, no. 10, pp. 1219-1232, 2013.

[12] A. Marino, Sugimoto, Mitsunobu, and Ouchi, et al., "Validating a notch filter for detection of targets at sea with ALOS-PALSAR data: Tokyo Bay," IEEE Journal of Selected Topics in Applied Earth Observation and Remote Sensing, vol. 7, no. 12, pp. 4907-4918, Dec. 2014.

[13] A. Marino and I. Hajnsek, "Statistical tests for a ship detector based on the polarimetric notch filter," IEEE Transactions on Geoscience and Remote Sensing., vol. 53, no. 8, pp. 4578-4595, Aug. 2015.

[14] G. Gao and G. Shi, "CFAR Ship Detection in Nonhomogeneous Sea Clutter Using Polarimetric SAR Data Based on the Notch Filter," IEEE Transactions on Geoscience and Remote Sensing., vol. 55, no. 8, pp. 4811-4824, Aug. 2017.

[15] L. M. Novak and M. C. Burl, "Optimal speckle reduction in polarimetric SAR imagery," IEEE Transactions on Aerospace and Electronic Systems., vol. 26, no. 2, pp. 293-305, Mar. 1990.

[16] L. M. Novak, M. C. Burl, and W.W. Irving. et al, "Optimal polarimetric processing for enhanced target detection," in Proc. Telesystems Conference Proceedings, Oct. 1991, pp. 69-75.

[17] R. Touzi, W M Boerner, J S Lee \& E. Lueneburg, "A review of polarimetry in the context of synthetic aperture radar: concepts and information extraction," Canadian Journal of Remote Sensing, vol. 30, no. 3, pp.380-407, 2004.

[18] A. Lopes and F. Sery, "Optimal speckle reduction for the product model in multilook polarimetric SAR imagery and the Wishart distribution," IEEE Transactions on Geoscience and Remote Sensing., vol. 35, no. 3, pp.
632-647, May.1997.

[19] G. Liu, S. Huang, and A. Torre, et al., "The multilook polarimetric whitening filter (MPWF) for intensity speckle reduction in polarimetric SAR images," IEEE transactions on geoscience and remote sensing., vol. 36, no. 3, pp. 1016-1020, May. 1998.

[20] R. Touzi, J. Hurley and P. W. Vachon, "Optimization of the Degree of Polarization for Enhanced Ship Detection Using Polarimetric RADARSAT-2," in IEEE Transactions on Geoscience and Remote Sensing, vol. 53, no. 10, pp. 5403-5424, Oct. 2015.

[21] R. Touzi and P.W. Vachon, "RCM Polarimetric SAR for Enhanced Ship Detection and Classification", Canadian Journal of Remote Sensing, Vol. 41, No. 5, pp 473-484, Feb. 2016.

[22] T. Liu, J. Zhang, G. Gao, J. Yang and A. Marino, "CFAR Ship Detection in Polarimetric Synthetic Aperture Radar Images Based on Whitening Filter," in IEEE Transactions on Geoscience and Remote Sensing. doi: 10.1109/TGRS.2019.2931353.

[23] D. Tao, S. N. Anfinsen and C. Brekke, "Robust CFAR Detector Based on Truncated Statistics in Multiple-Target Situations," IEEE Transactions on Geoscience and Remote Sensing., vol. 54, no. 1, pp. 117-134, Jan. 2016.

[24] H. M. Finn and R. S. Johnson, "Adaptive detection mode with threshold control as a function of spatially sampled clutter-level estimates," RCA Rev., vol. 29, pp. 414-464, Sep. 1968.

[25] V. Hansen and J. Sawyers, "Detectability loss due to "Greatest Of" se-lection in a cell-averaging CFAR," IEEE Trans. Aerosp. Electron. Syst., vol. AES-16, no. 1, pp. 115-118, Jan. 1980.

[26] G. Trunk, "Range resolution of targets using automatic detectors," IEEE Trans. Aerosp. Electron. Syst., vol. AES-14, no. 5, pp. 750-755, Sep. 1978.

[27] M. Smith and P. Varshney, "Intelligent CFAR processor based on data variability," IEEE Trans. Aerosp. Electron. Syst., vol. 36, no. 3, pp. 837-847, Jul. 2000.

[28] H. Rohling, "Radar CFAR thresholding in clutter and multiple target situations," IEEE Trans. Aerosp. Electron. Syst., vol. AES-19, no. 4, pp. 608-621, Jul. 1983.

[29] S. Blake, "OS-CFAR theory for multiple targets and nonuniform clutter," IEEE Trans. Aerosp. Electron. Syst., vol. 24, no. 6, pp. 785-790, Nov. 1988

[30] P. Gandhi and S. Kassam, "Analysis of CFAR processors in homoge-neous background," IEEE Trans. Aerosp. Electron. Syst., vol. 24, no. 4, pp. 427-445, Jul. 1988.

[31] J. Rickard and G. Dillard, "Adaptive detection algorithms for multiple-target situations," IEEE Trans. Aerosp. Electron. Syst., vol. AES-13, no. 4, pp. 338-343, Jul. 1977.

[32] M. Barkat, S. Himonas, and P. Varshney, "CFAR detection for multi-ple target situations," Proc. Inst. Elect. Eng.-F Radar Signal Process., vol. 136, no. 5, pp. 193-209, Oct. 1989.

[33] S. Khan, and R. Guida, "On Fractional Moments of Multilook Polarimetric Whitening Filter for Polarimetric SAR Data," IEEE Transactions on Geoscience and Remote Sensing., vol. 52, no. 6, pp. 3502-3512, 2014.

[34] A. Lopes and F. Sery, "Optimal speckle reduction for the product model in multilook polarimetric SAR imagery and the Wishart distribution," in IEEE Transactions on Geoscience and Remote Sensing, vol. 35, no. 3, pp. 632-647, May 1997.

[35] G. M.Tallis, "Elliptical and Radial Truncation in Normal Populations." The Annals of Mathematical Statistics, vol. 34, no. 3, 1963, pp. 940-944. JSTOR, www.jstor.org/stable/2238475.

[36] D. Tao, A. P. Doulgeris and C. Brekke, "A Segmentation-Based CFAR Detection Algorithm Using Truncated Statistics," in IEEE Transactions on Geoscience and Remote Sensing, vol. 54, no. 5, pp. 2887-2898, May 2016.

[37] Wishart, John. "The Generalised Product Moment Distribution in Samples from a Normal Multivariate Population." Biometrika, vol. 20A, no. $1 / 2$, pp. 32-52, 1928.

[38] Goodman, N. R. "Statistical Analysis Based on a Certain Multivariate Complex Gaussian Distribution (An Introduction)." The Annals of Mathematical Statistics, vol. 34, no. 1, pp. 152-177,1963.

[39] M. Abramowitz, I. A. Stegun, Handbook of Mathematical Functions With Formulas, Graphs, and Mathematical Tables, Dover Publications Inc., NYC, USA, 2001.

[40] JamolPender, The truncated normal distribution: Applications to queues with impatient customers, Operations Research Letters, Volume 43, Issue 1, January 2015, Pages 40-45

[41] I. B. Aban, M. M. Meerschaert, and A. K. Panorska, "Parameter estimation for the truncated pareto distribution," Journal of the American Statistical Assoc., vol. 101, no. 473, pp. 270-277, March 2006. 
[42] G.M. Tallis, The moment generating function of the truncated multi-normal distribution, J. R. Stat. Soc. Ser. B Stat. Methodol. 23 (1) (1961) 223-229

[43] G.M. Tallis, Plane truncation in normal populations, J. R. Stat. Soc. Ser. B Stat. Methodol. (ISSN: 00359246) 27 (2) (1965) 301-307

[44] N.L. Johnson, S. Kotz, N. Balakrishnan, Continuous Univariate Distributions, Vol. 1, John Wiley \& Sons, 1994.

[45] Juan C.Arismendi, Simon Broda. Multivariate elliptical truncated moments. Journal of Multivariate Analysis. Volume 157, May 2017, Pages $29-44$

[46] Johnson RW, Kliche DV, Smith PL. 2011. Comparison of estimators for parameters of gamma distributions with left-truncated samples. J. Appl. Meteorol. Climatol. 50: 296-310.

[47] Johnson, R. W., Kliche, D. V. and Smith, P. L. (2014), Maximum likelihood estimation of gamma parameters for coarsely binned and truncated raindrop size data. Q.J.R. Meteorol. Soc., 140: 1245-1256.

[48] N. Giri, "On the complex analogues of T2- and R2-tests," Ann. Math.Stat., vol. 36, no. 2, pp. 664-670, Apr. 1965.

[49] K.O. Geddes, M.L. Glasser, R.A. Moore and T.C. Scott, Evaluation of Classes of Definite Integrals Involving Elementary Functions via Differentiation of Special Functions, AAECC (Applicable Algebra in Engineering, Communication and Computing), vol. 1, (1990), pp. 149-165

[50] Liu, T., Cui, H., Xi, Z. et al. Novel estimators of equivalent number of looks in polarimetric SAR imagery based on sub-matrices, Sci. China Inf. Sci. (2016) 59: 062309. https://doi.org/10.1007/s11432-015-5480-x

[51] Crisp, D.J., 2004, The State-of-the Art in Ship Detection in Synthetic Aperture Radar Imagery. Research Report DSTO-RR-0272, Defence Science \& Technology Organisation, Australia.

[52] D. Velotto, C. Bentes, B. Tings and S. Lehner, "First Comparison of Sentinel-1 and TerraSAR-X Data in the Framework of Maritime Targets Detection: South Italy Case," in IEEE Journal of Oceanic Engineering, vol. 41, no. 4, pp. 993-1006, Oct. 2016.

[53] J. Martín-de-Nicolás, P. Jarabo-Amores, N. del-Rey-Maestre, P. Gómez-del-Hoyo and J. L. Bárcena-Humanes, "Robustness of a Generalized Gamma CFAR ship detector applied to TerraSAR-X and Sentinel-1 images," IEEE EUROCON 2015 - International Conference on Computer as a Tool (EUROCON), Salamanca, 2015, pp. 1-6.

[54] B. Barboy, A. Lomes and E. Perkalski, "Cell-averaging CFAR for multiple-target situations," in IEE Proceedings F - Communications, Radar and Signal Processing, vol. 133, no. 2, pp. 176-186, April 1986.

[55] G. Gao, L. Liu, L. Zhao, G. Shi and G. Kuang, "An Adaptive and Fast CFAR Algorithm Based on Automatic Censoring for Target Detection in High-Resolution SAR Images," in IEEE Transactions on Geoscience and Remote Sensing, vol. 47, no. 6, pp. 1685-1697, June 2009.

[56] Y. Cui, G. Zhou, J. Yang and Y. Yamaguchi, "On the Iterative Censoring for Target Detection in SAR Images," in IEEE Geoscience and Remote Sensing Letters, vol. 8, no. 4, pp. 641-645, July 2011.

[57] S. Song, B. Xu and J. Yang, "Ship Detection in Polarimetric SAR Images via Variational Bayesian Inference," in IEEE Journal of Selected Topics in Applied Earth Observations and Remote Sensing, vol. 10, no. 6, pp. 2819-2829, June 2017.

[58] C. Liu and C. H. Gierull, "A New Application for PolSAR Imagery in the Field of Moving Target Indication/Ship Detection," in IEEE Transactions on Geoscience and Remote Sensing, vol. 45, no. 11, pp. 3426-3436, Nov. 2007.

[59] S. Kullback and R. A. Leibler, "On information and sufficiency," Ann. Math. Statist., vol. 22, no. 1, pp. 79-86, 1951.

[60]M. Ester, "A Density-based Algorithm for Discover erring Clusters in Large Spatial Databases with Noise," In Proc. 2nd International Conference on Know ledge Discovery and Data Mining. Aug. 1996.

[61]R. L. Paes, J. A. Lorenzzetti, and D. F. M. Gherardi, "Ship detection using TerraSAR-X images in the campo basin (Brazil)," IEEE Geosci. Remote Sens. Lett., vol. 7, no. 3, pp. 545-548, Jul. 2010.

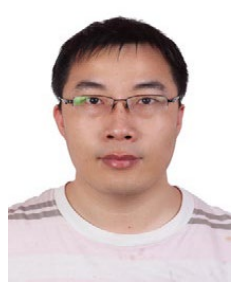

Tao Liu received the B.S. degree in communication engineering and the Ph.D. degree in information and communication engineering, all from the National University of Defense Technology (NUDT), Changsha, China, in 2001 and 2007 respectively. In 2000, he was the Outstanding Communist
Youth League member of China. Since 2007, he has been with the School of Electronic Engineering, Naval University of Engineering, where he is currently a Professor. In 2009 he received the Award of Excellent Doctor Thesis of Chinese Army. Since 2019 he is a committee member in Radar Branch of the Chinese Institute of Electronics (CIE).

He has authored over 50 journal papers and three books. His research interests include statistical theory of radar polarization, polarization information processing, synthetic aperture radar (SAR) automatic target recognition, statistical modeling of SAR image, SAR ship detection, InSAR (interferometric SAR), SAR ground moving target indication and Artificial Intelligence(AI).

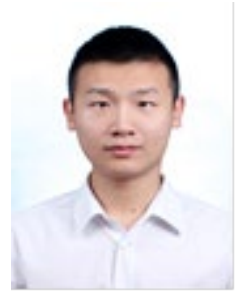

Ziyuan Yang received the B.S. degree in radar engineering from the Naval University of Engineering (NUE), Wuhan, China, in 2019 . Now he is studying for a doctor's degree in information and communication engineering.

His research interests include radar polarization information process and electronic warfare system modeling and SAR ground moving target indication.

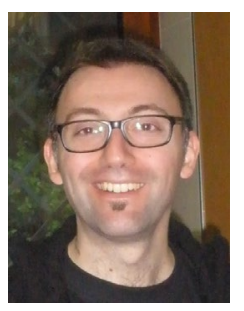

Armando Marino (M'2011) received the M.Sc. degree in telecommunication engineering from the Universita' di Napoli "Federico II," Naples, Italy, in 2006. In 2006, he joined the High Frequency and Radar Systems Department, German Aerospace Centre, Oberpfaffenhofen, Germany, where he developed his M.Sc. thesis. He received the $\mathrm{Ph} . \mathrm{D}$. degree in polarimetric SAR interferometry from the School of Geosciences, University of Edinburgh, Edinburgh, U.K., in 2011. From March 2011 to October 2011, he was with the University of Alicante, Institute of Computing Research, Spain. From December 2011 to May 2015, he was a Postdoctoral Researcher and Lecturer with ETH Zurich, Institute of Environmental Engineering, Switzerland. From June 2015, he was a Lecturer with the School of Engineering and Innovation, Open University, Milton Keynes, U.K. Since May 2018 he is a Senior Lecturer (Assistant Professor) at the University of Stirling, Faculty of Natural Sciences, Stirling, UK.

Dr. Marino's Ph.D. thesis received the "Best Ph.D. Thesis 2011 " by the Remote Sensing and Photogrammetry Society and the "Outstanding Ph.D. Thesis" by Springer Verlag, which published the thesis in 2012.

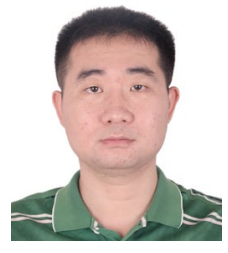

Gui Gao (M'09) received the B.S. in information engineering, the M.S. and Ph.D. degrees in remote sensing information processing from National University of Defense Technology (NUDT), Changsha, China, in 2002, 2003 and 2007, respectively. From 2007, he joined the Faculty of Information Engineering, School of Electronic Science and Engineering, NUDT, as an 
associate professor. From 2017, he was with Faculty of Geosciences and Environmental Engineering, Southwest Jiaotong University, Chengdu, China, where he is currently a professor. He is also a guest professor of the College of Traffic Engineering, Hunan University of Technology, Zhuzhou, China. He has authored over 100 journal and conference papers and has written four books and an English chapter. His current research interests include radar signal processing, InSAR (interferometric SAR), target detection, marine environment, and SAR GMTI (ground moving target indication).

Dr. Gao is a member of the IEEE Geoscience and Remote Sensing Society, the Applied Computational Electromagnetics Society, and a senior member of the Chinese Institute of Electronics (CIE), and a Dominant Member of Young Scientist Forum of CIE. He received the Award of Excellent Master Thesis of Hunan Province in 2006, the Award of Excellent Doctor Thesis of Chinese Army in 2008, Awards of Outstanding Young People in NUDT and Hunan Province of China in 2014 and 2016, a first Class Prize of Chinese Military Science and Technology Progress Award, and the Award of Natural Science in Hunan Province. He was also selected as Young Talents of Hunan in 2016 and supported by the Excellent Young People Science Foundation of the National Natural Science Foundation of China. He is the Leader Guest Editor of the International Journal of Antenna and Propagation, the Guest Editor of the Remote Sensing, the Associate Editor and Leader Guest Editor of the IEEE Journal of Selected Topics in Applied Earth Observations and Remote Sensing, and on the Editorial Board of the Chinese Journal of Radars. He was also the Co-Chairman of several conferences in the field of remote sensing. He was the Excellent Reviewer for the journal of Xi'an Jiaotong University in 2013.

Jian Yang (M'03, SM' 04) received the B.S. and M.S. degrees from Northwestern

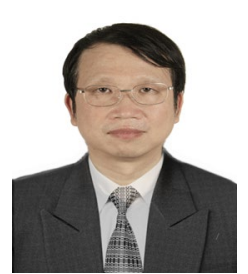
Polytechnical University, Xian, China, in 1985 and 1990, respectively, and the Ph.D. degree from Niigata University, Niigata, Japan, in 1999. In 1985, he joined the Department of Applied Mathematics, Northwestern Polytechnical University. From 1999 to 2000, he was an Assistant Professor with Niigata University. In April 2000, he joined the Department of Electronic Engineering, Tsinghua University, Beijing, China, and he was promoted to a full Professor in 2002. He has published more than 300 papers and received many awards. His research interesting areas include radar polarimetry, feature extraction, target detection and target classification. 